\title{
Research Paper \\ Analysis of Gender Differences in Time Use Among Iranian Older Adults
}

\author{
Nasibeh Zanjari ${ }^{1} \odot{ }^{*}$ Rasoul Sadeghi $^{2,3}$, Ahmad Delbari ${ }^{1}$
}

1. Iranian Research Centre on Aging, University of Social Welfare and Rehabilitation Sciences, Tehran, Iran.

2. Department of Demography, Faculty of Social Sciences, University of Tehran, Tehran, Iran.

3. National Population Studies \& Comprehensive Management Institute, Tehran, Iran.

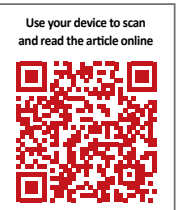

dtation Zanjari N, Sadeghi R, Delbari A. [Analysis of Gender Differences in Time Use Among Iranian Older Adults (Persian)]. Salmand: Iranian Journal of Ageing. 2019; 13(Special Issue):588-603. : https://doi.org/10.32598/SIJA.13.Special-Issue.588

https://doi.org/10.32598/SIJA.13.Special-Issue.588

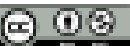

Received: 05 Jun 2018 Accepted: 08 Dec 2018 Available Online: 10 Mar 2019

Key words:

Gender difference,

Time use patterns, Social class, leisure activities, Older adults, Iran

\begin{abstract}
Objectives Time use patterns affect humans' health and quality of life. Different social contexts such as gender roles impact on time use patterns in each society. The purpose of this article is to examine gender differences in time use patterns of Iranian older adults.

Methods \& Materials The method of this study is the secondary analysis of the Iranian time use national survey data. A multistage stratified random sampling method was used to recruit the study samples. The study data were collected by completing the study questionnaires of 4285 Iranian elderly 60 years and older who were living in urban areas.

Results In this study, $52 \%$ of the subjects were male and $48 \%$ female. The Mean \pm SD age of males respondents was $69.2 \pm 7.8$ years and for females, it was $68.7 \pm 7.5$ years. About $24.5 \%$ of elderly women were living alone compared with $6.6 \%$ of men. Also, $5.5 \%$ of the elderly men and $6.8 \%$ of elderly women were disabled and need caregivers. In addition, the findings showed that elderly men allocated more time to paid works ( 2 hours per day for men in comparison to 6 minutes per day for women). In contrast, elderly women allocated more time to unpaid works such as caregiving and services to family ( 4.7 hours per day for women in comparison to 1.7 hours per day for men). Also, elderly men participated more in active leisure than women $(P<0.05)$. Although the sleep time for the elderly women was more than that in elderly men, the time for eating, as well as self-care for men was more than women. According to the multivariate analysis, the gender differences in time use allocation were due to socioeconomic gender differences. Conclusion Gender differences in time use allocation are due to socio-cultural and economic factors. Thus, we suggest that appropriate social welfare policies be designed to reduce gender differences in time use patterns by taking into account these important factors.
\end{abstract}

\section{Extended Abstract}

\section{Objectives}

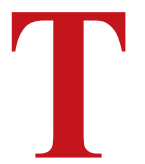

he time use pattern affects the health and quality of life of the elderly $[1,2]$. These patterns are influenced by community context and socio-demographic factors such as gender. In this regard, this study aimed to examine the gender differences in time use among Iranian elderly people.

\section{Methods \& Materials}

In this study, we conducted a secondary analysis of the 2014 national time use survey data. The samples were 4285 older people aged $\geq 60$ years living in urban areas of Iran. They were selected using a multistage stratified random

\section{* Corresponding Author:}

Rasoul Sadeghi, PhD.

Address: Department of Demography, Faculty of Social Sciences, University of Tehran, Tehran, Iran

Tel: +98 (21) 61117884

E-mail: rassadeghi@ut.ac.ir 
sampling method. The study data were collected by filling questionnaires and holding interviews based on time allocation technique. In analyzing data, three concepts of "primary activity", "participation rate", and "average time" were considered. Primary activity refers to the major activity that is performed at a specific time. For example, if the subject was watching TV while eating, one of these activities is considered a primary activity. The participation rate is the percentage of people involved in the activity, and the average time is the time calculated for people who do and those who do not perform an activity. The obtained data were analyzed by multiple regression analysis in SPSS V. 20.

\section{Results}

In this study, $52 \%$ of the subjects were male and $48 \%$ were female. The mean \pm SD age of men was $69.2 \pm 7.8$ years and for women, it was $68.7 \pm 7.5$ years. About $6.6 \%$ of men and $24.5 \%$ of women were living alone. Moreover, $5.5 \%$ of men and $6.8 \%$ of women had disabilities and needed health care. As seen in Figure 1, elderly men devoted more time to paid works ( $2 \mathrm{~h}$ per day vs. 6 min per day in women), while elderly women mostly performed unpaid works such as providing care and services to family members $(4.7 \mathrm{~h}$ per day vs. $1.7 \mathrm{~h}$ per day in men). Furthermore, elderly men performed leisure activities significantly more than elderly women $(\mathrm{P}<0.05)$. The sleep time of elderly women was more than that of men, while the time spent on eating and drinking as well as the time for personal care activities in elderly men was more than that in elderly women.

According to Figure 2, the participation rate of elderly men and women in paid works, unpaid works, religious activities, and community volunteering was noticeably different. About $27 \%$ of elderly men participated in paid works, while for elderly women, the participation rate was $5 \%$. On the other hand, $93 \%$ of elderly women participated in unpaid works (e.g. providing care and services to family members) and for men, it was $69 \%$. Moreover, $72 \%$ of elderly women participated in voluntary activities in the community, compared to $63 \%$ of older men. Furthermore, the participation rate of elderly men and women in religious activities were $87 \%$ and $84 \%$, respectively. Therefore, economic activities are more common in elderly men while social activities are more common in elderly women.

We used multiple regression analysis to examine the relationship of gender with time use patterns by controlling socio-demographic variables. Results showed that gender differences in using time are mostly due to differences in the socio-economic status of men and women and traditional patterns of gender roles.

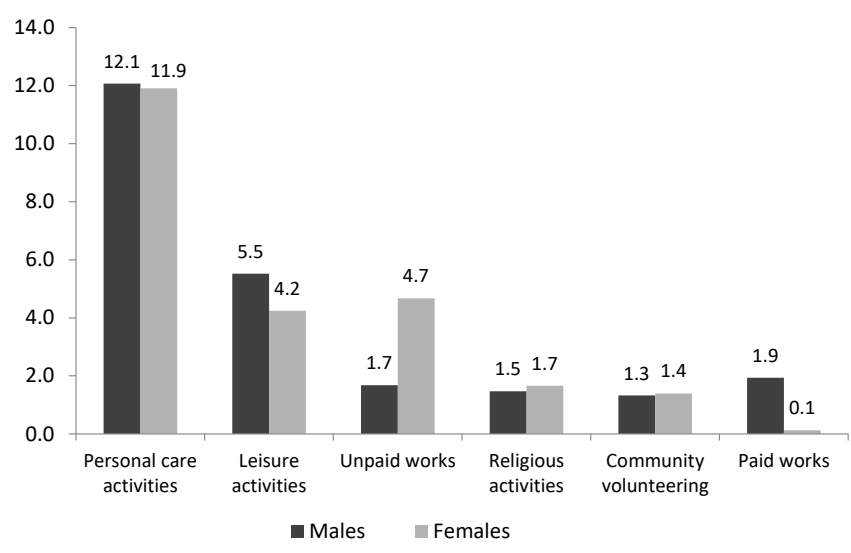

Figure 1. Time use patterns of the study subjects based on gender

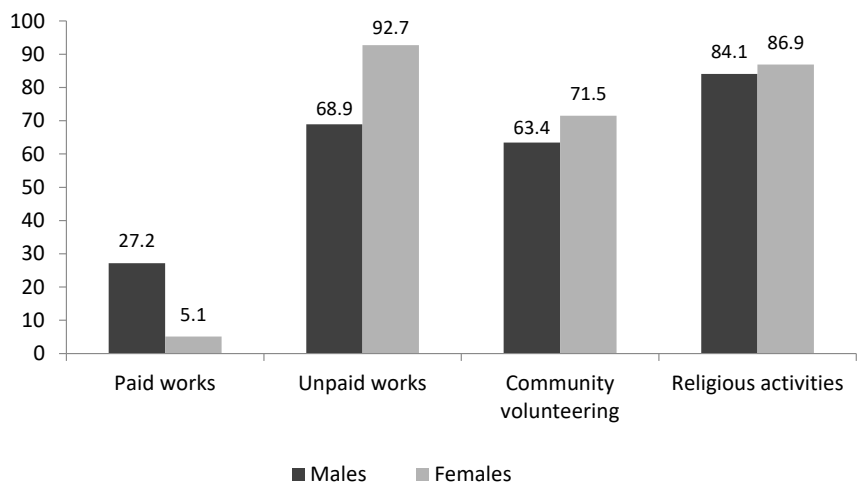

Figure 2. The participation rate of the study subjects in different activities 


\section{Conclusion}

Time is one of the human resources that is affected by gender. In other words, gender influences time use patterns and time allocation in different ways. Gender differences in patterns of time use are mainly due to gender inequalities in economic and social opportunities, culture and social norms, gender-based division of labor, and the roles of women at home. Hence, welfare policies and programs need to be designed and implemented with the aim of reducing gender differences in old age. Considering the limitations of this study, it is suggested that in future studies, the role of the elderly's time use patterns in their health and well-being be considered, too. Also, considering that this study focused on urban areas, it is recommended that further studies be conducted on time use patterns of elderly women and men in rural areas.

\section{Ethical Considerations}

Compliance with ethical guidelines

This study has been approved by Iranian Research Center on Aging and the Research Ethics Committee of University of Social Welfare and Rehabilitation Sciences (code: IR.USWR.REC.1395.350).

Funding

This study has been supported by Iranian Research Center on Aging, University of Social Welfare and Rehabilitation Sciences (code: 1493).

\section{Authors' contributions}

Conceptualization and methodology: Nasibeh Zanjari and Rasoul Sadeghi; Formal analysis: Rasoul Sadeghi; Investigation, resources, and draft preparation: Nasibeh Zanjari; Editing: Ahmad Delbari; Supervision, and project administration: Nasibeh Zanjari; and Funding acquisition: Nasibeh Zanjari and Ahmad Delbari.

\section{Conflicts of interest}

The authors declared no conflict of interest.

\section{Acknowledgments}

The authors would like to thank Iran Statistical Center and Statistical Research and Training Center for providing the study data. 


\title{
تحليل تفاوتهاى جنسيتى در الكَوهاى كَذران وقت سالمندان ايرانى
}

\author{
نسيبه زنجرى' ه. • رسول صادقى "rr م. احمد دلبرى'
}

ا - مركز تحقيقات سالمئدى، دانشخاه علوم بهزيستى و توانبخشى، تهران، ايران.

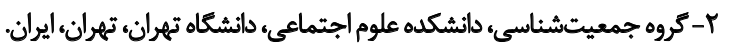

r- مؤسسه مطالعات و مديريت جامع و تخصصى جمعيت كشور، تهران، ايران.

\begin{abstract}
حكיد

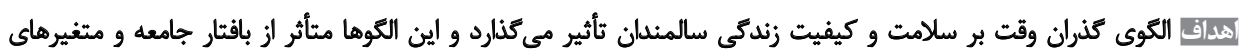

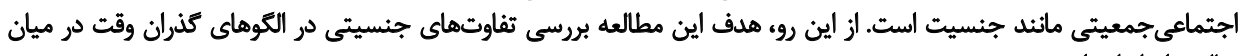
سالمندان ايراني استي.

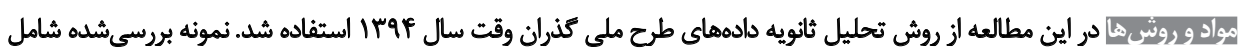

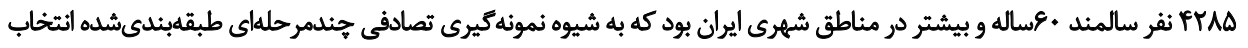

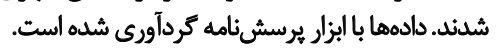

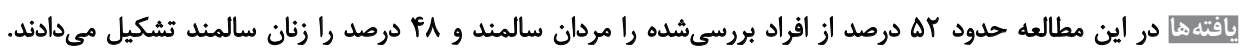

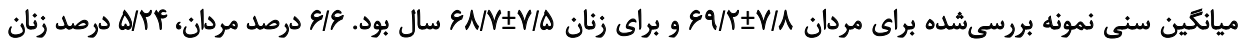

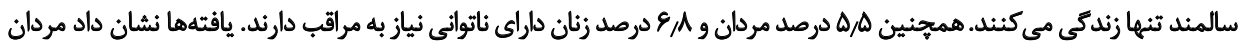

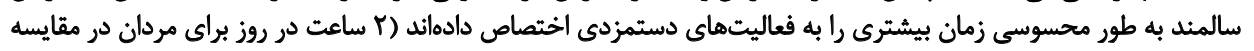

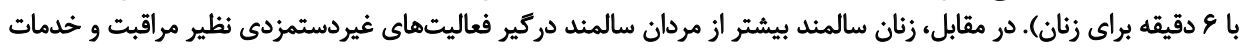

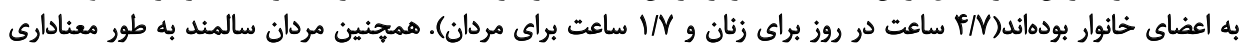

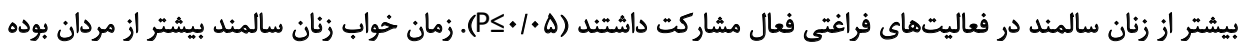

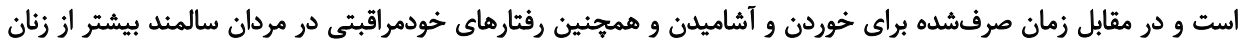

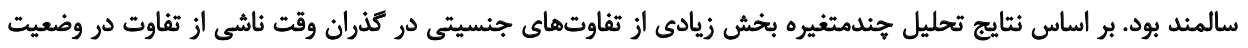

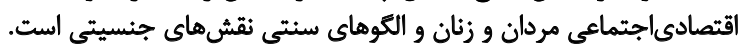

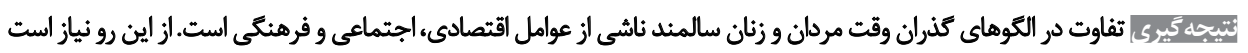

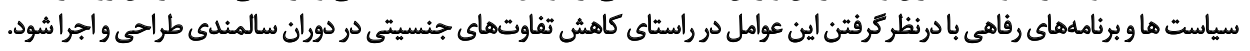

تاريخ دريافت: هاخرداد Trav

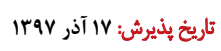
تاريخ انتشار: 199 اسفند

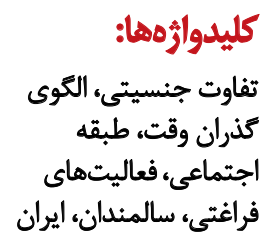

كليدوارهها:

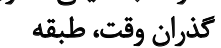

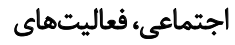
فراغتى، سالمندان، ايران فيتهاي

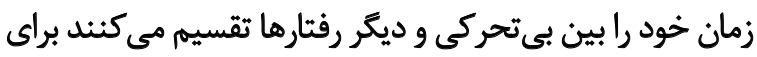

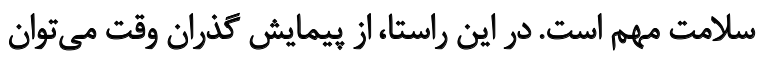

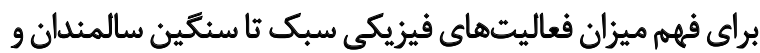

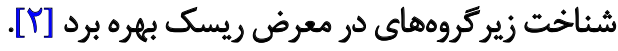

به علاوه، الكوهاى كذران وقت در دوران سالمندى بارزتر از

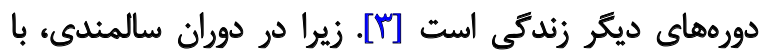

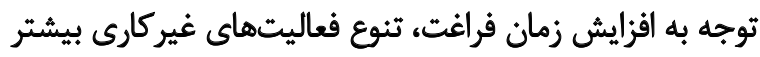

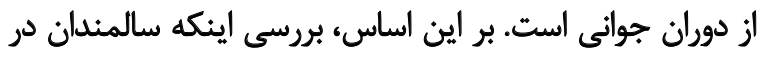

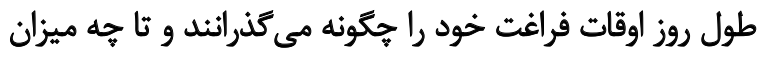

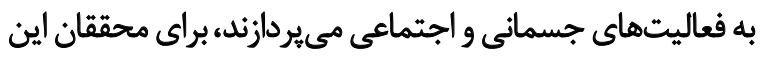

dolio

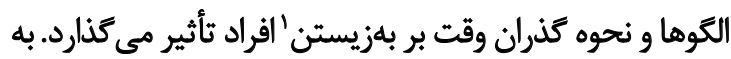

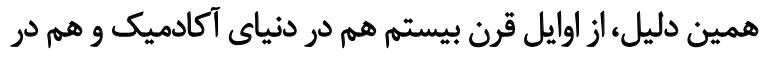

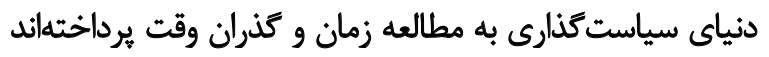

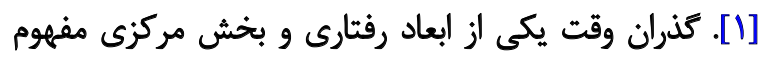

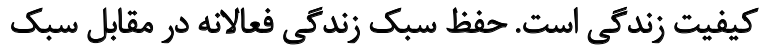

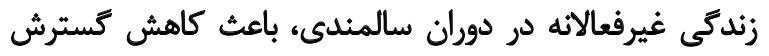

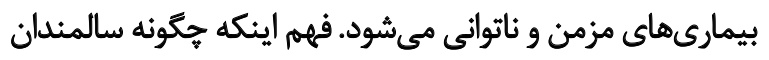

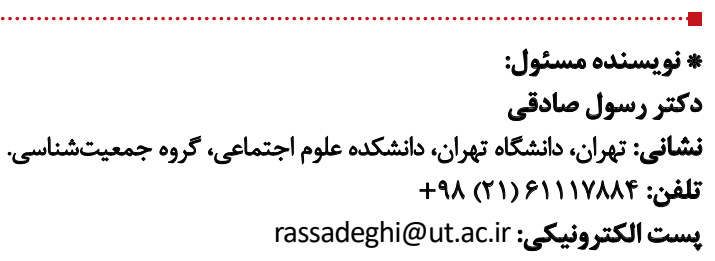


به كار با درآمد و كار خانكى وجود دارد [N]].

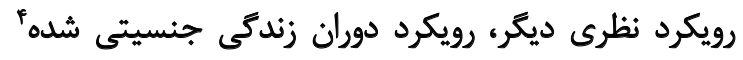

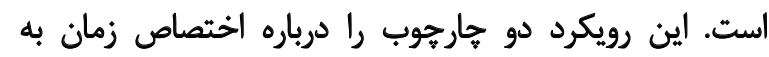

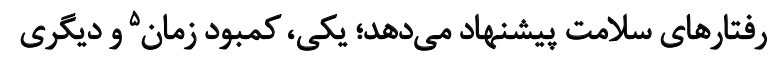

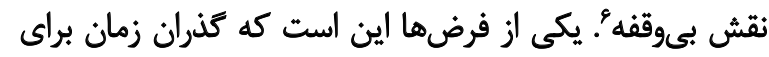

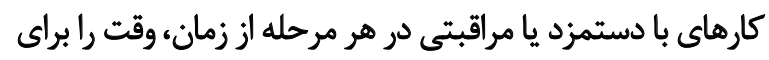

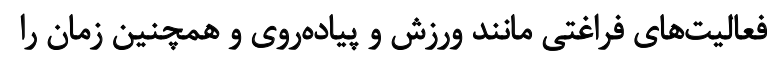

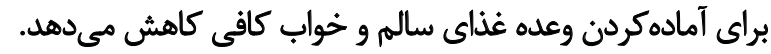

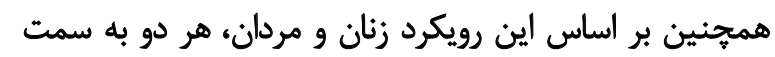

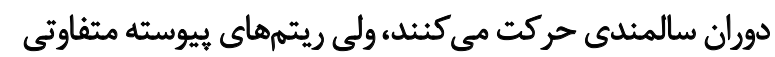

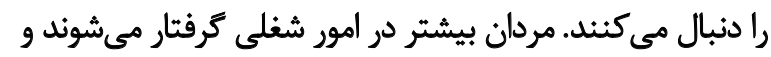

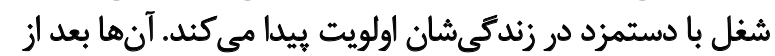

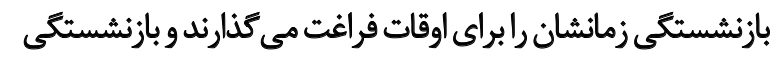

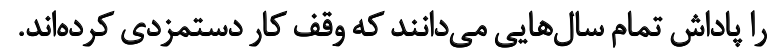

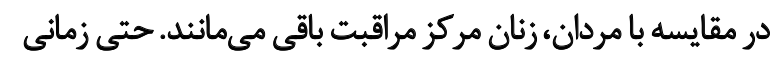

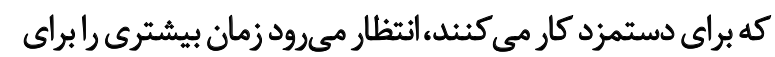

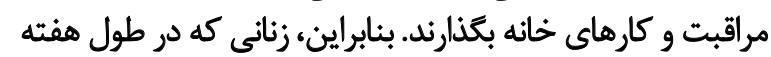

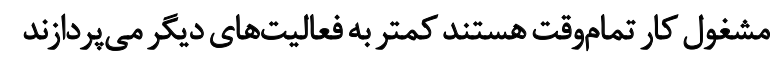

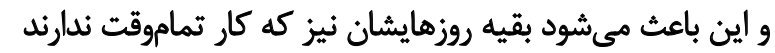

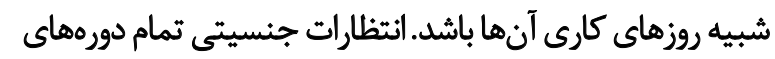

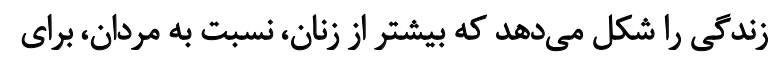

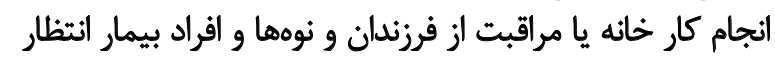

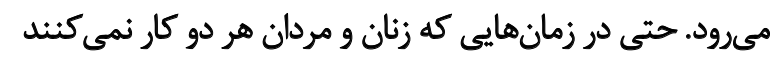

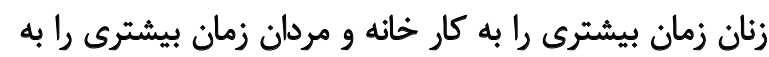

فراغت اختصاص مى دهند [9].

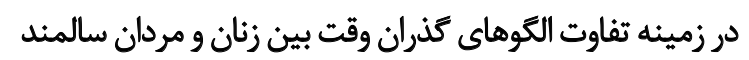

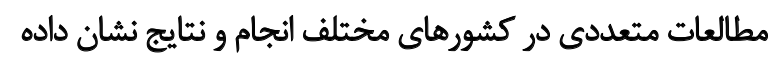

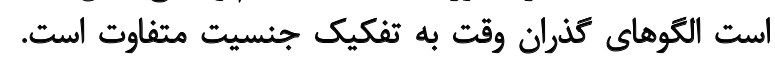

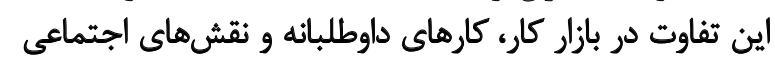

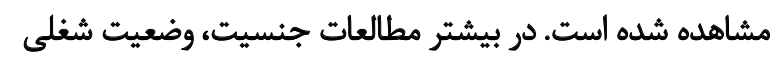

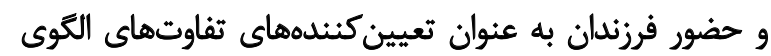

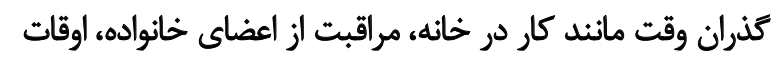

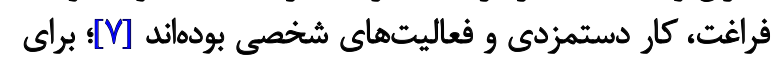

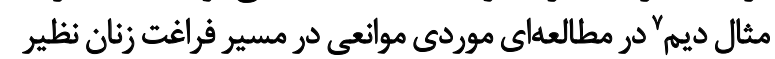

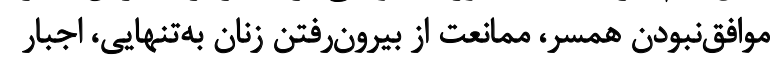

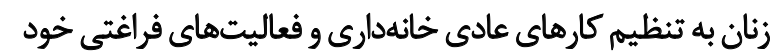

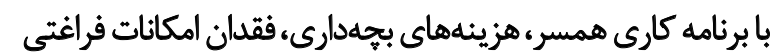

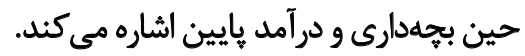
بستر بدرسالارانه و مؤلفههاى اقتصادى و اجتماعى نظير

4. Gendered life course

5. Time scarcity

6. Roleless role

7. Deem
عرصه و سياست كذارى در زمينه سالمندى سالم كمك كننده

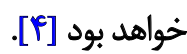

در كذران وقت، نابرابرى اجتماعى در اشكال مختلف آن بروز

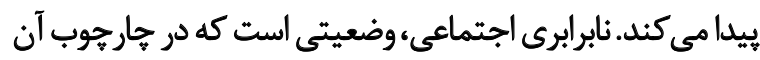

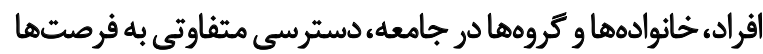

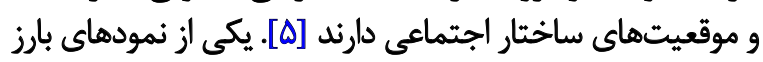

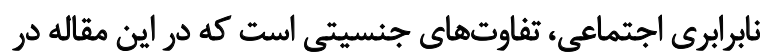

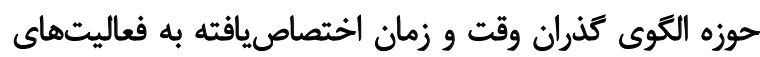
مختلف زندكى بروسى مكى وقود.

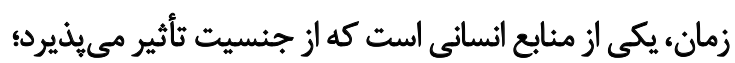

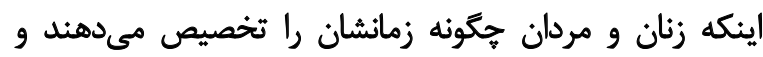

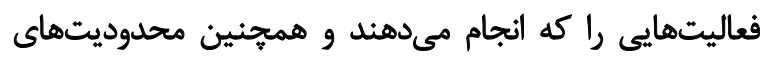

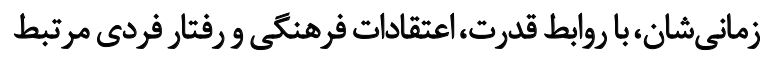

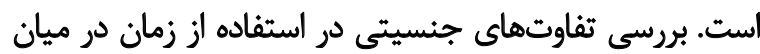

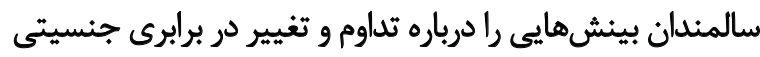

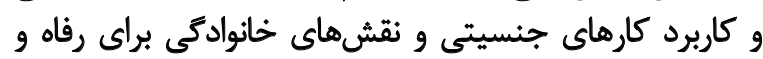

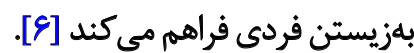

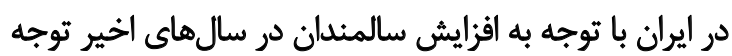

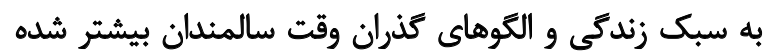

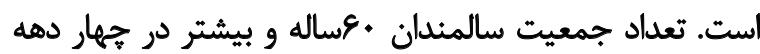

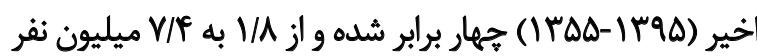

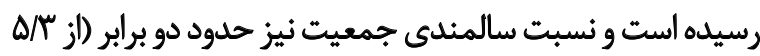

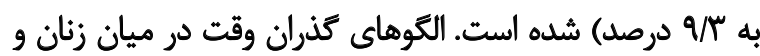

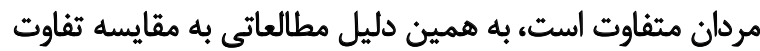

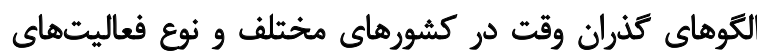

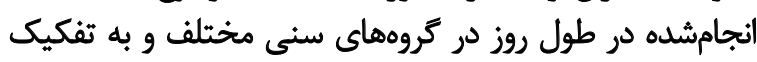

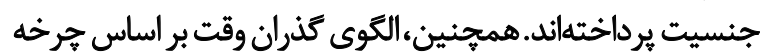

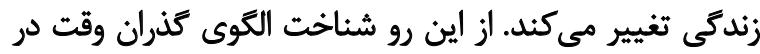

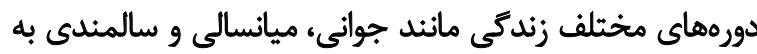

$$
\text { تفكيك جنسيت مخهم است. }
$$

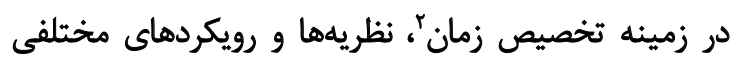

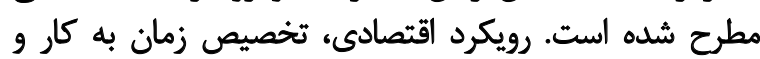

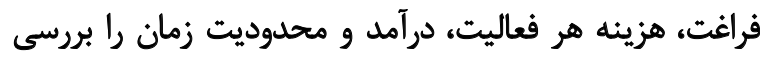

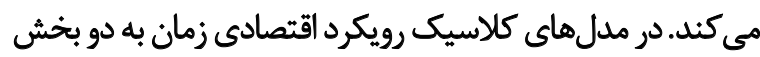

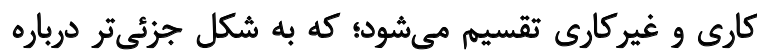

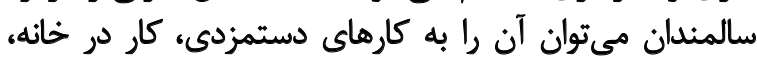

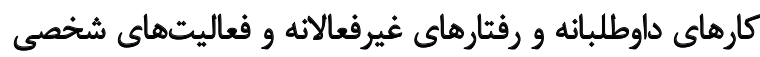

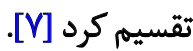

در نظريههاى جديد اقتصادى نظير نظريه عملكرد نون توليد خانوار"

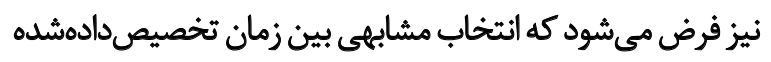

2. Time allocation

3. Household production function 
نظامهاى رفاه و سياست عمومى [عץ] تأكيد داشتهاند. همجنين، در حيطه الكوى كذران وقت در دوران سالمندى،

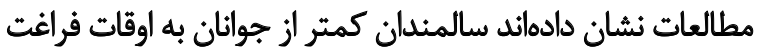

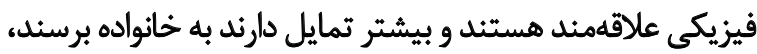

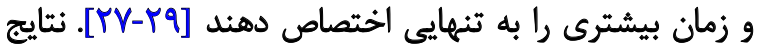

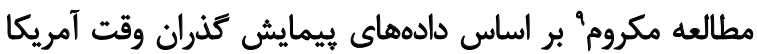

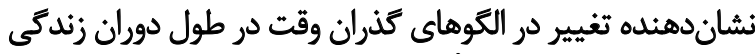

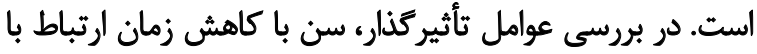

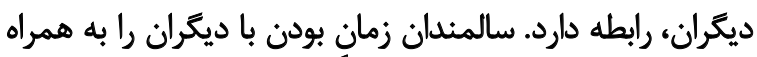

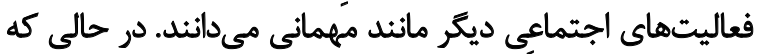

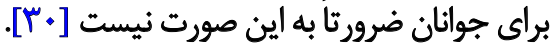

در مطالعه الكوهاي كذران وقت در ميان سالمندان كشورهاي

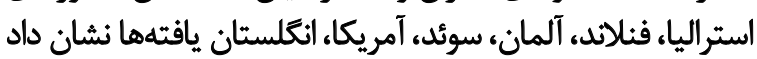

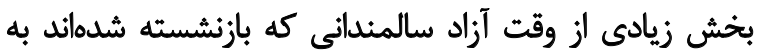

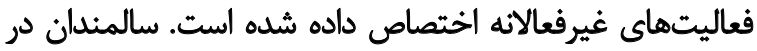

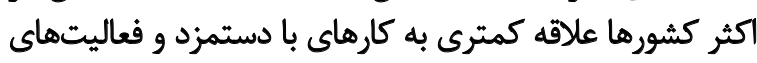

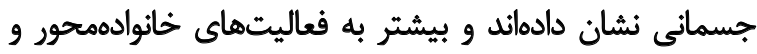

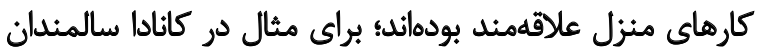

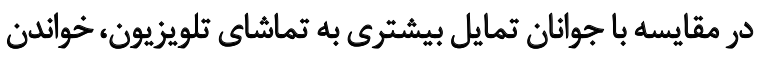

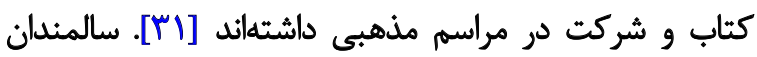

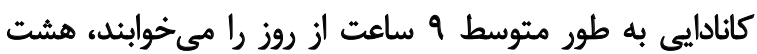

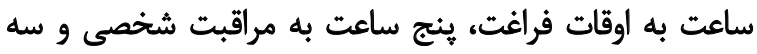

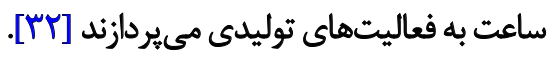

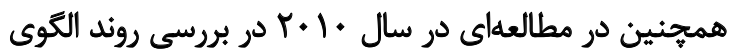

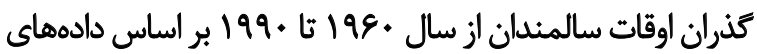

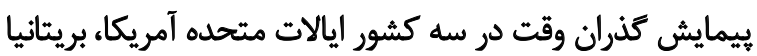

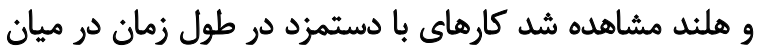

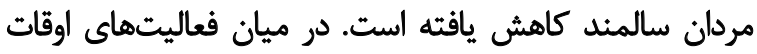

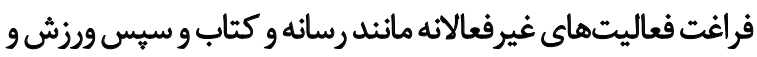

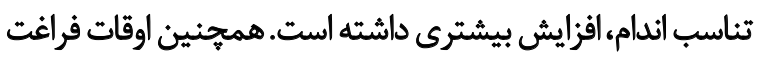

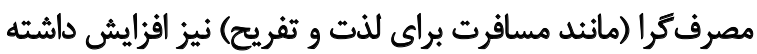

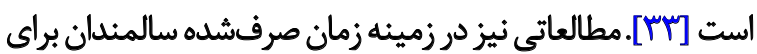

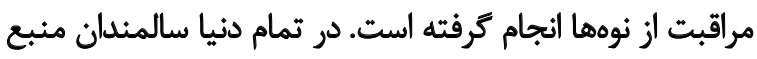

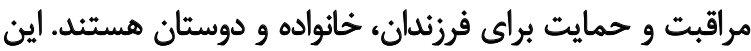

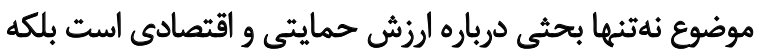

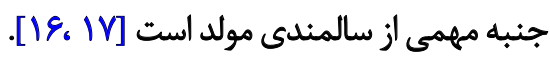

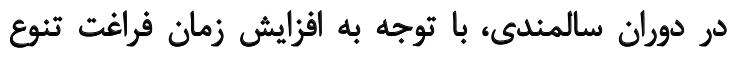

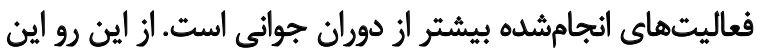

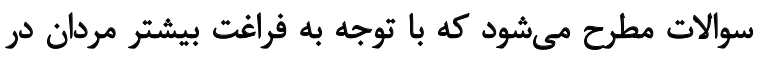

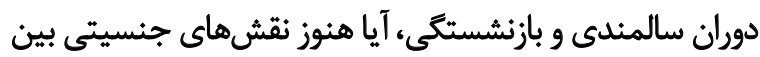
زنان و مردان وجود دارد يا خير؟ مردان و زنان سالمند زمانشان ران ران
اينها عواملى هستند كه تا حدودى دليل مشاركت كم زنان

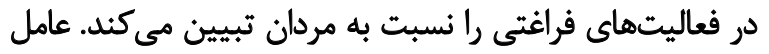

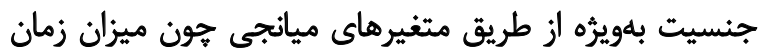

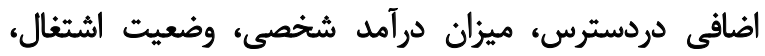

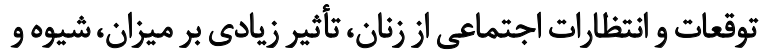

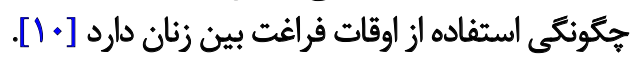

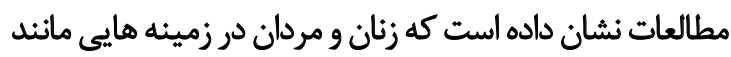

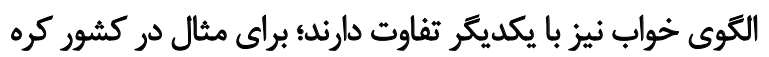

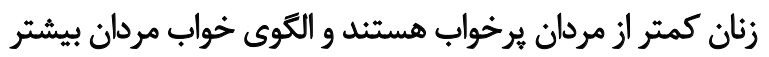

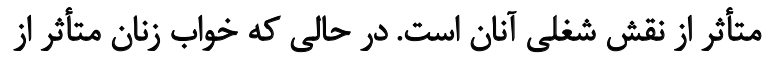

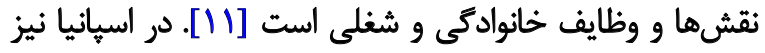

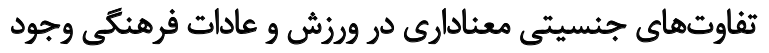

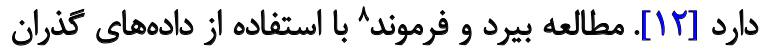

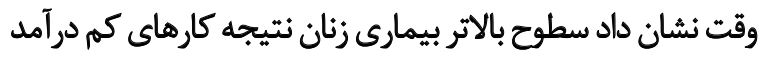

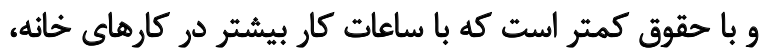

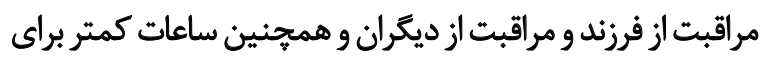

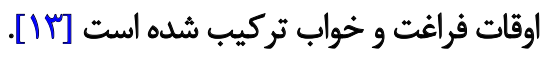
علاوه بر اوقات فراغت اين تفاوت جنسيتى در كارهاى

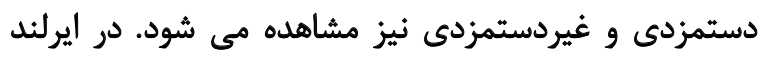

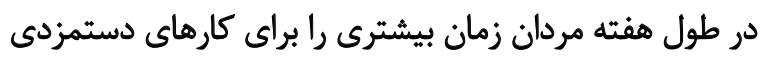

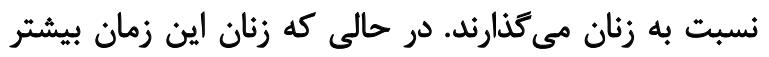

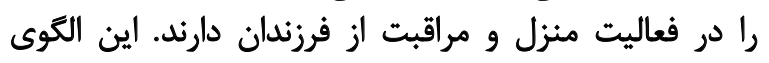

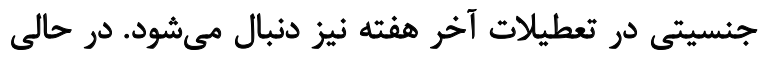

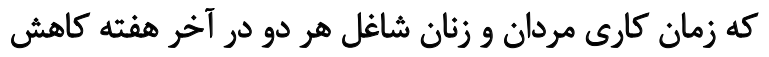

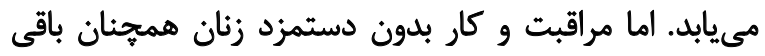

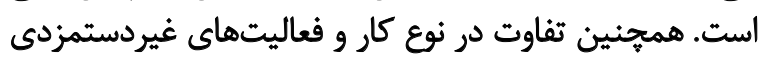

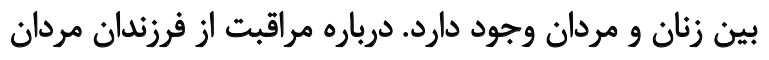

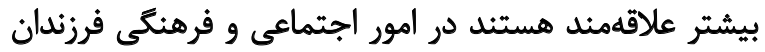

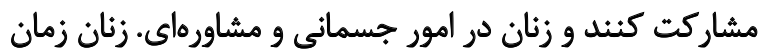

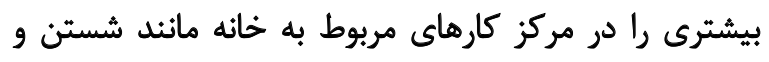

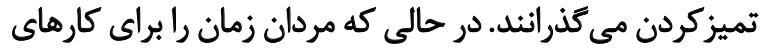

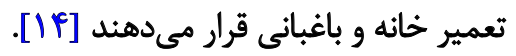

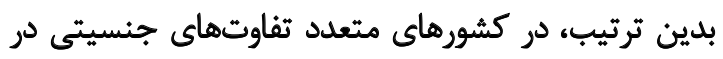

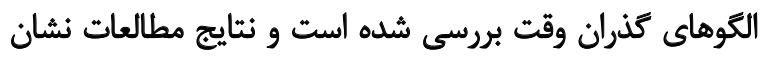

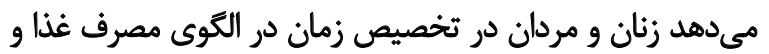

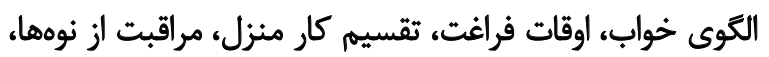

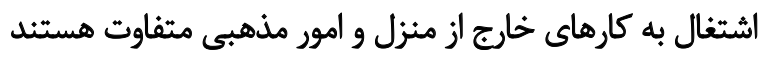

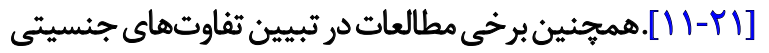

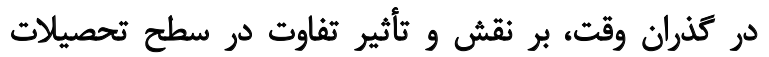

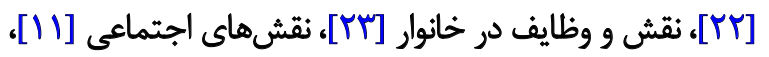

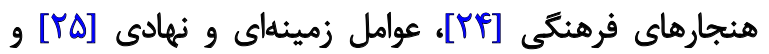


شامل دو گروه اشتغال رسمى / دولتي" و اشتغال غيررسمى|

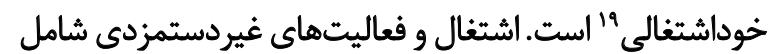

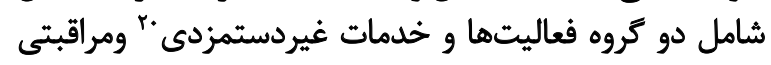

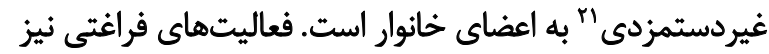

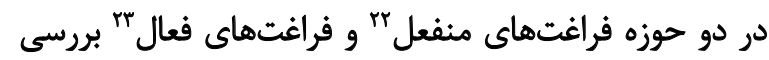

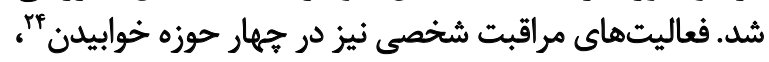

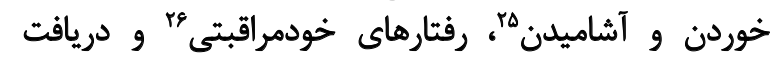

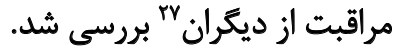

متغير مستقل در اين تحقيق، جنسيت است كه در مقوله

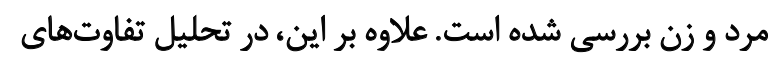

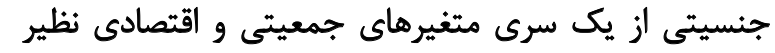

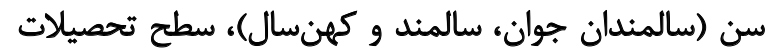

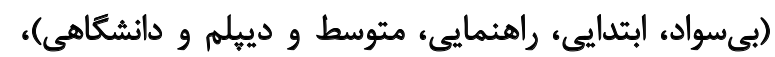

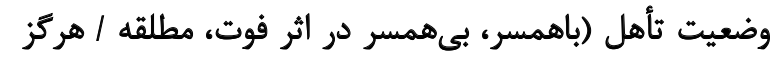

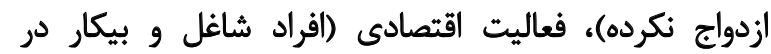

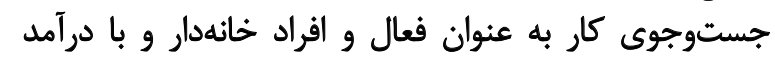

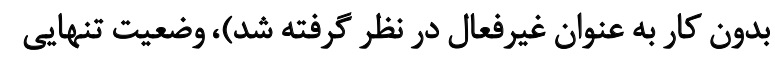

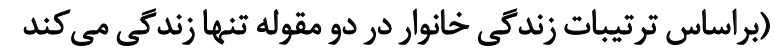

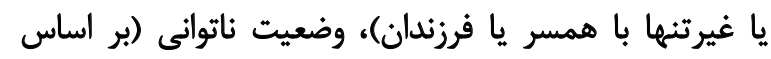

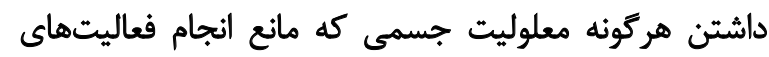

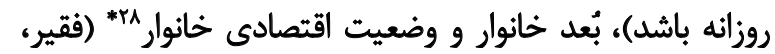
متوسط و مرفه) به عنوان متغيرهاى كنترل استفاده شده است.

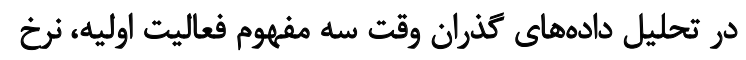

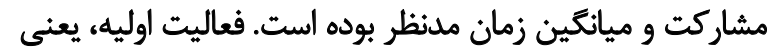

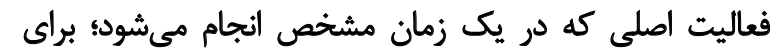

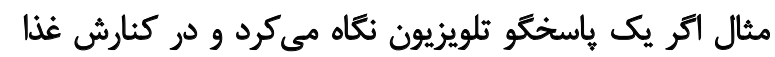

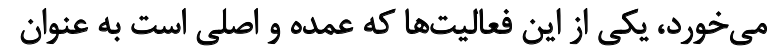

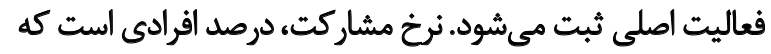

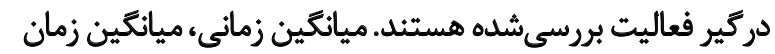
محاسبهشده براى افرادى است كه يك فعاليت را انجام مى دهنيند

\section{Formal employment}

19. Informal \& Self-employment

20. Unpaid services to family

21. Unpaid care giving to family

22. Passive leisure

23. Active leisure

24. Sleep

25. Eating and drinking

26. Self-care behaviors

27. Receiving care

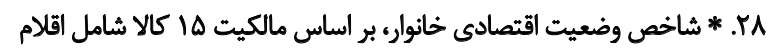

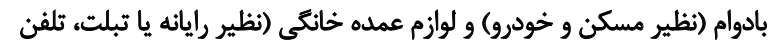

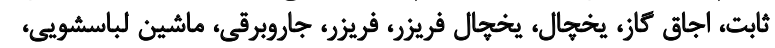

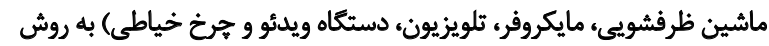

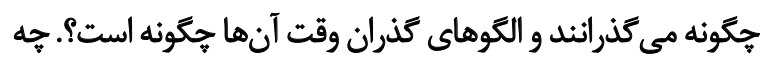

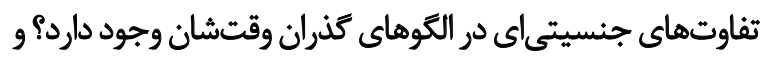

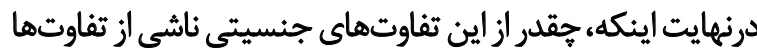

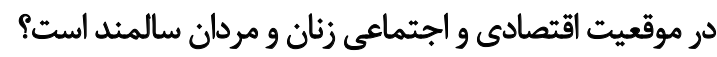

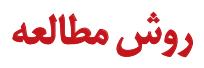

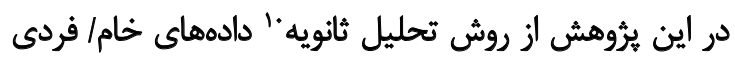

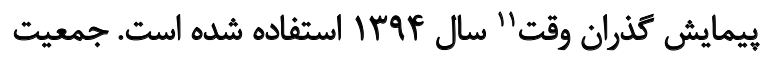

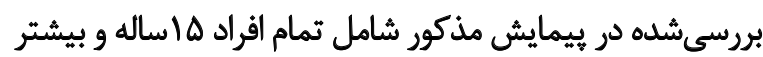

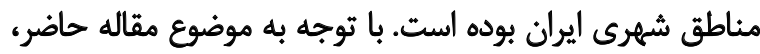

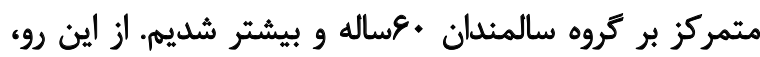

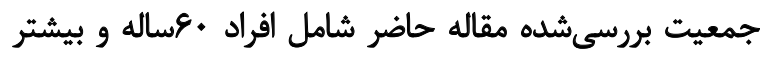

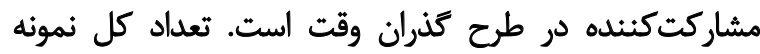

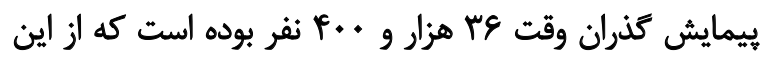

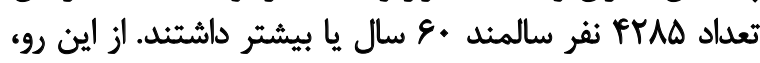

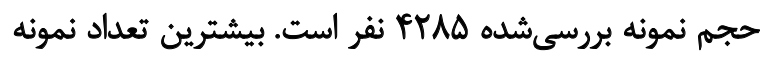

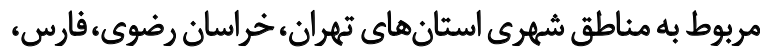

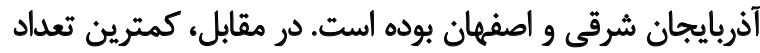

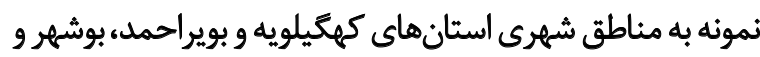
خراسان شمالى تعلق داشته است.

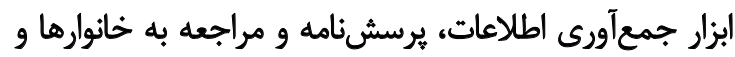

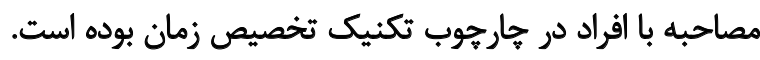

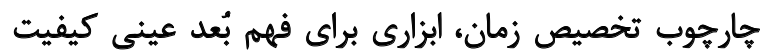

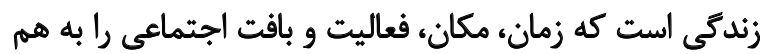

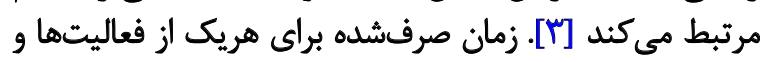

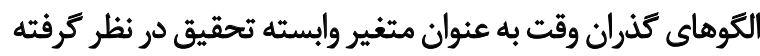

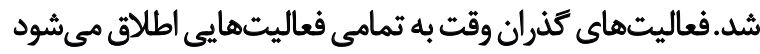

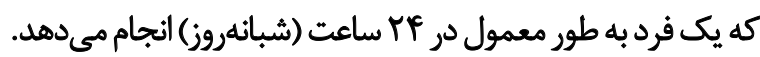
در اين يروهش، مشاركت و زمان صرفشده سالمندان در

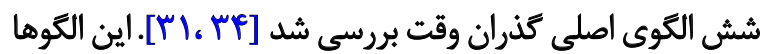

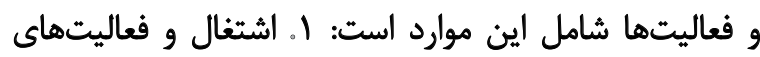

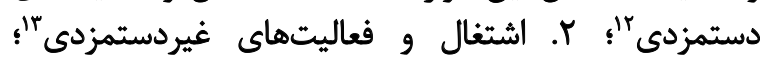

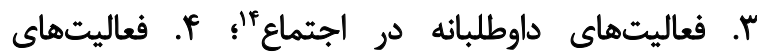

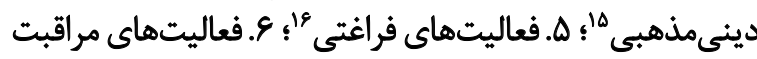

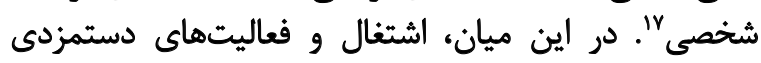

10. Secondary analysis

11. Time use

12. Paid works

13. Unpaid works

14. Community Volunteering

15. Religious activities

16. Leisure activities

17. Personal care activities 
بوده است. در حالي كه ب M درصد مردان سالمند تحصيلات دييلم و بيشتر داشتهاند اين رقم براي زنان سالمند ه در در صد بوده است. به لحاظ وضعيت زناشويى، M د درصد مردان سالمند در زمان

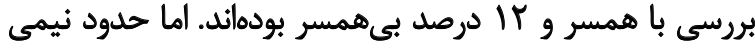

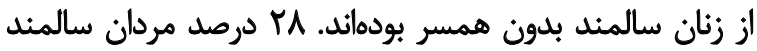

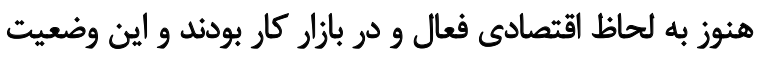

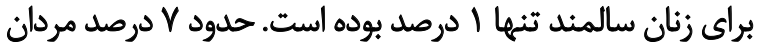

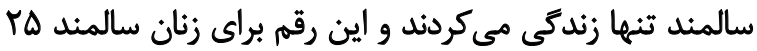

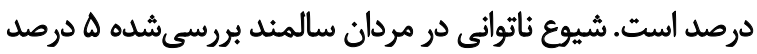

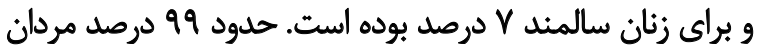

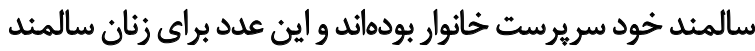

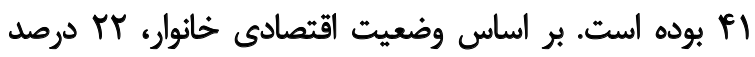

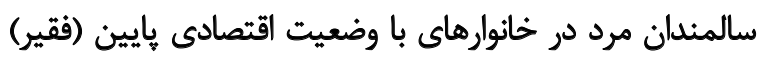

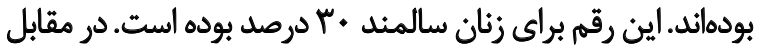

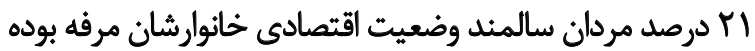

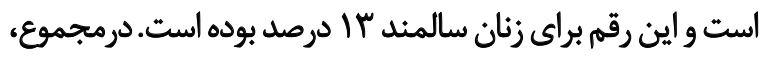

و آنهايي كه انجام نمي دهند. تجزيه و تحليل دادهها با استفاده

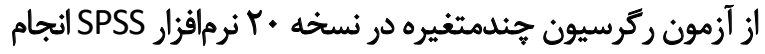
شده است. أسمن

Ladị

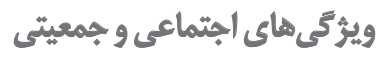

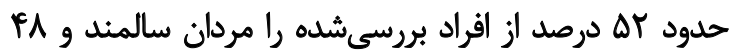

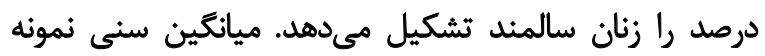

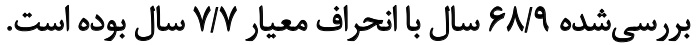
بررسي ويرّكَّىهاي اقتصادي، اجتماعي و جمعيتي سالمندان

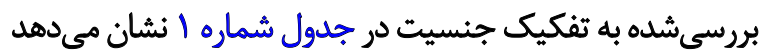

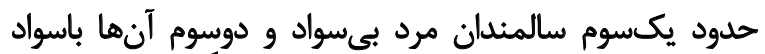

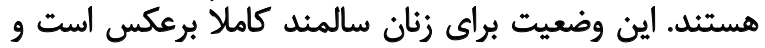

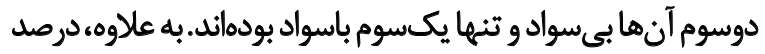
زنان سالمند با سطوح بالاى تحصيلى بسيار كمتر از مردان سالمند

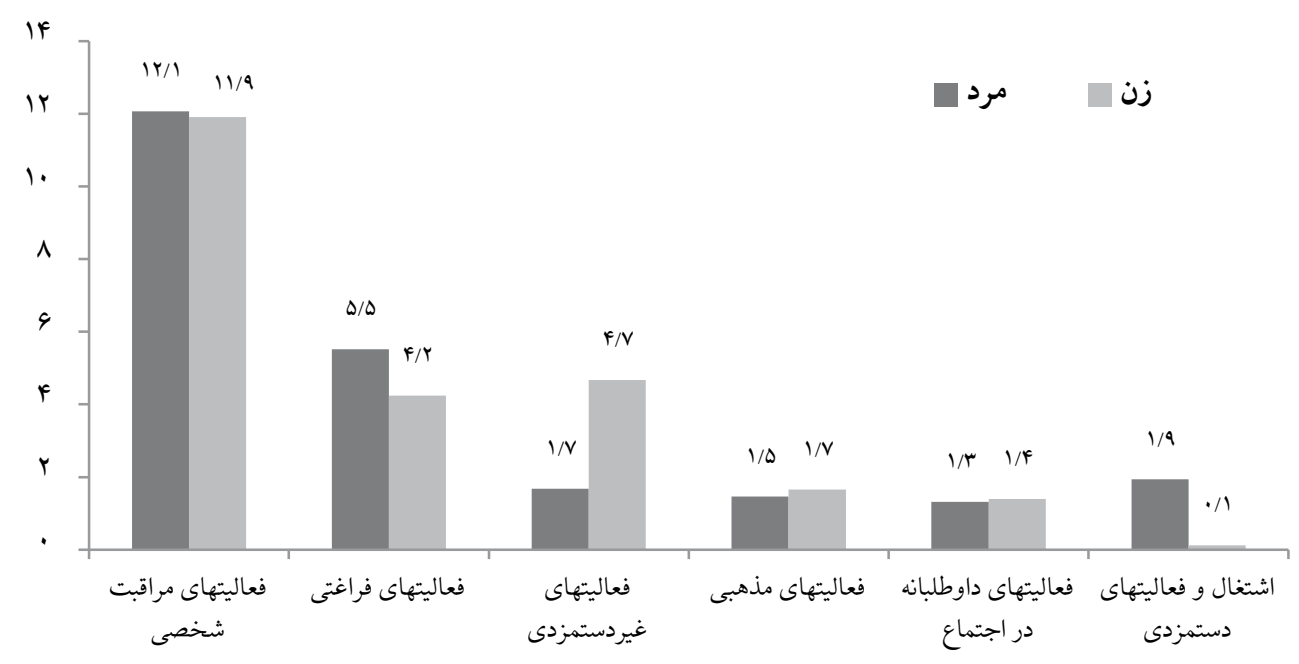

تصوير ا. الكوهاى كذران وقت سالمندان بررسىشده به تفكيك جنسيت (ميانكّين ساعات در روز)

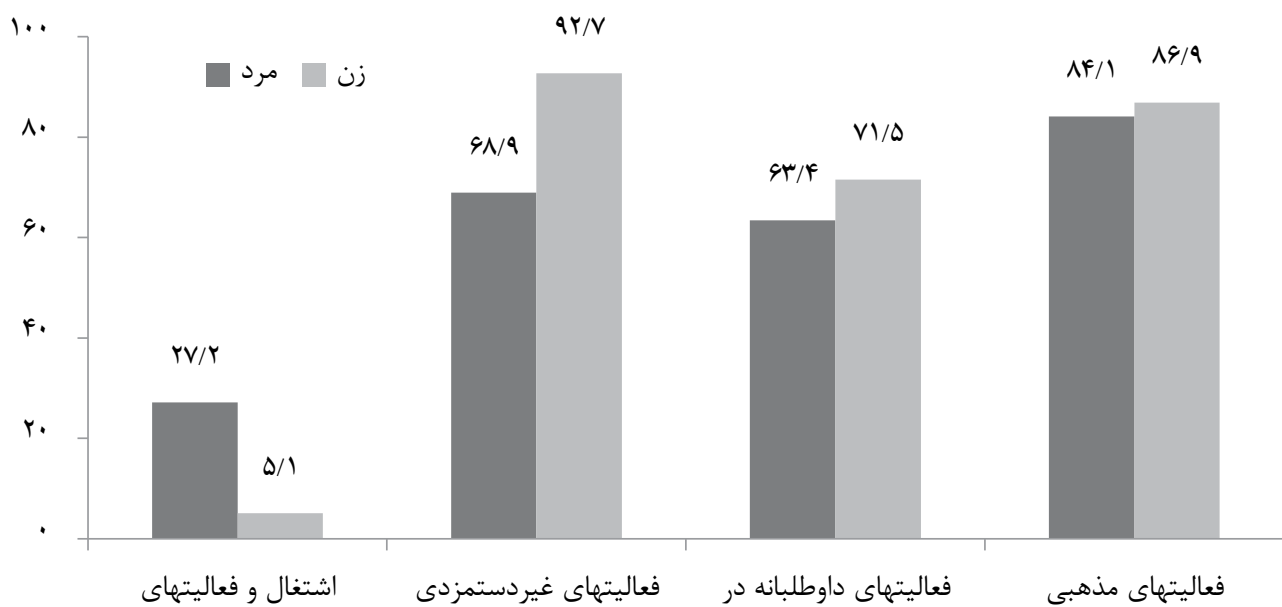


جدول ا. ويرٔكى هاي اجتماعى و جمعيتى مردان و زنان سالمند، ITFF

\begin{tabular}{|c|c|c|c|c|c|}
\hline \multicolumn{2}{|c|}{ زنان } & \multicolumn{2}{|c|}{ مردان } & \multirow{2}{*}{\multicolumn{2}{|c|}{ ويزوكى هاى اجتماعىجمعيتى }} \\
\hline درصد & تعداد & ورد & تعداد & & \\
\hline$\Delta q / T^{m}$ & $|r|$ & $\Delta N N$ & ImIA & سالمند جوان (•\&-89) & \\
\hline$r q / 9$ & 81. & TNI & शri & (V.-Vq) & \\
\hline $1 . / 1$ & $M M$ & $\mid m / 1$ & rqه & كمنسال (1+1+) & سن \\
\hline \multicolumn{2}{|c|}{ ENV(V/D) } & \multicolumn{2}{|c|}{$g a / T(V / A)$} & ميانكين سنى (انحراف معيار) & \\
\hline$g / 8$ & irqu & $m \in /$ & VEA & بيىسواد & \multirow{2}{*}{ وضع سواد } \\
\hline$r g / F$ & ner & gA/q & Ifva & باسواد & \\
\hline $94 / 8$ & iras & $m / 1$ & VEA & 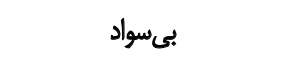 & \multirow{5}{*}{ سطح تحصيلات } \\
\hline$r / \Delta$ & pA. & $M T / q$ & Vra & ابتدايى & \\
\hline$H / V$ & 90 & $1 . / r$ & MTA & راهنمايى & \\
\hline$\Delta / \&$ & 110 & $i r / r$ & rqe & هتوسطه و دييلم & \\
\hline$r / 8$ & هr & $9 / 8$ & riv & دانشكاهى & \\
\hline ar & $1 . A r$ & NA & IQY & با همسر & \multirow{3}{*}{ وضعيت تأهل } \\
\hline$p e / F$ & $q \cdot v$ & $1 . / \pi$ & mTr & بيوه & \\
\hline$r / \Delta$ & ar & $1 / 1$ & ro & مطلقه - م م & \\
\hline Q⿻ & $r+1 r$ & $V / \Lambda$ & 1819 & غيرفعال & \multirow{2}{*}{ وضعيت فعاليت اقتصادى } \\
\hline $1 / \%$ & rq & rV/a & ETD & فعال & \\
\hline$V \Delta / \Delta$ & IDF. & $9 \% / F$ & $r+q$ & غيرتنها & \multirow{2}{*}{ وضعيت تنهايى } \\
\hline Tr/s & 0.1 & 818 & ifs & تنه & \\
\hline $9 \% / r$ & $19 \cdot r$ & $97 / \Delta$ & MIMI & 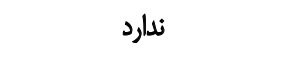 & \multirow{2}{*}{ وضعيت ناتوائى } \\
\hline $8 / 1$ & ITA & $\Delta / \Delta$ & $i m$ & مارد & \\
\hline$P_{1}$ & Are & QN/A & MIV & سريرست خانوار & \multirow{4}{*}{ رابطهل با سريرست خاثوار } \\
\hline$\Delta V / \Delta$ & 1.01 &.$/ 1$ & r & همسر سريرست & \\
\hline $9 / 8$ & imf & $1 / 1$ & me & هير و مادر سريرست & \\
\hline 1 & r. & - & 1 & ساير & \\
\hline$r e / \Delta$ & Q.1 & $8 / 8$ & IFA & 1 & \multirow{5}{*}{ بُعد خانوار } \\
\hline$M e / 8$ & $V \cdot V$ & rg/D & Ar. & r & \\
\hline $19 / 9$ & $f \cdot r$ & re/8 & $\Delta Q 1$ & $r$ & \\
\hline$r+/ q$ & fre & $r \cdot / r$ & gra & f & \\
\hline \multicolumn{2}{|c|}{$r / \Delta(1 / T)$} & \multicolumn{2}{|c|}{$m / \circ(1 / m)$} & ميانكين (انحراف معيار) & \\
\hline$r q / 1$ & $8 \cdot 1$ & $M / N$ & FAY & فقير & \multirow{4}{*}{ وضعيت اقتصادى خانوار } \\
\hline$\Delta V / \Delta$ & $11 n^{f}$ & $\Delta E / N$ & irrr & متوسط & \\
\hline$I T / N$ & roq & MVE & FAD & مرفه & \\
\hline $1 \ldots$ & $r+P_{1}$ & 1.0 & MMPF & مونه & \\
\hline
\end{tabular}


معنى، زنبودن اثرى منفى بر اشتغال دستمزدى دارد. در مدل

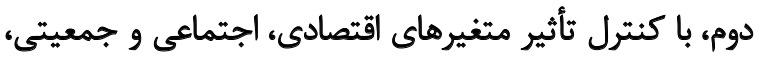

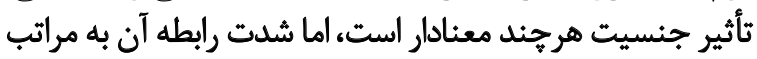

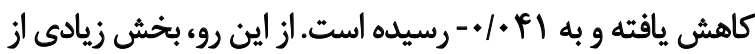

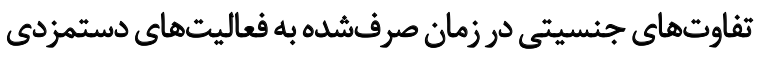

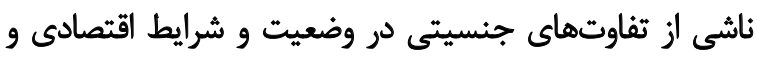

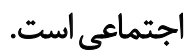

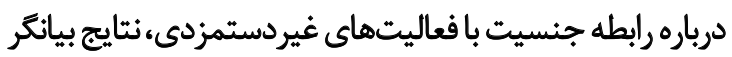

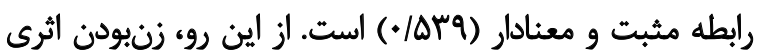

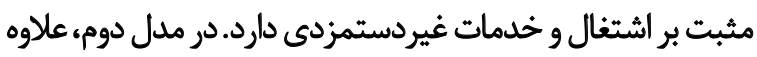

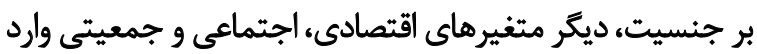

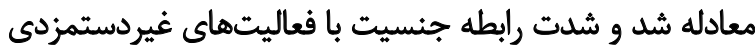

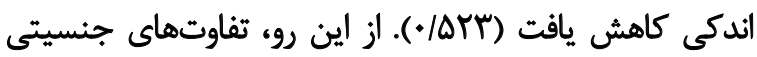

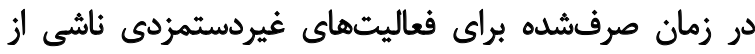

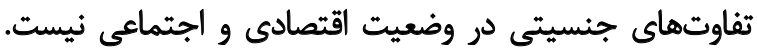

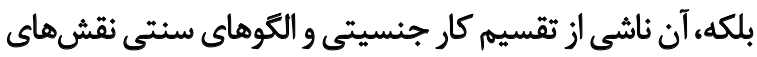

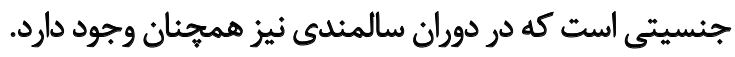

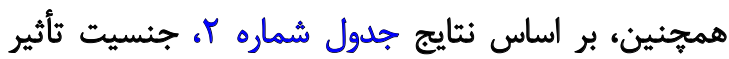

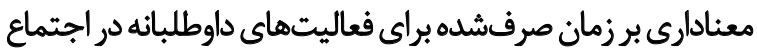

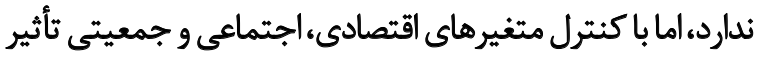

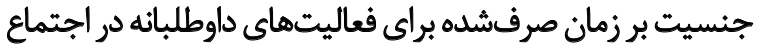

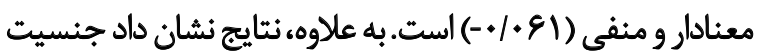

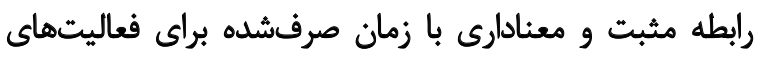

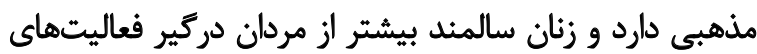

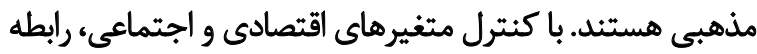

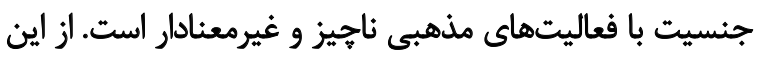

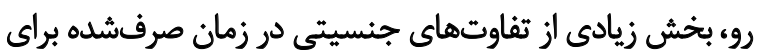

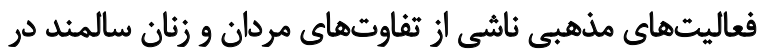

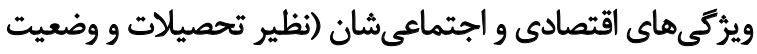

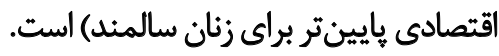

جنسيت رابطه منفى و معنادارى با زمان صرفشده براي

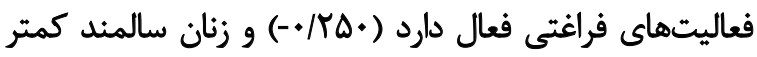

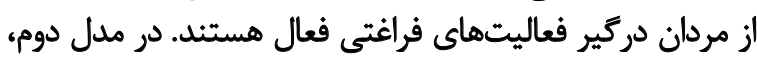

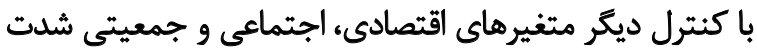

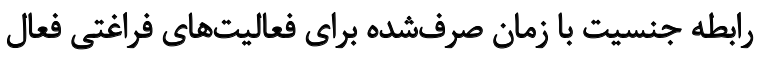

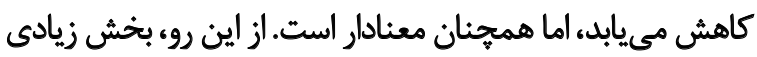

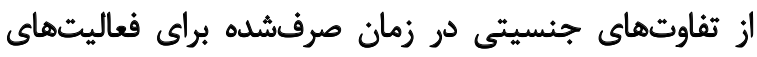

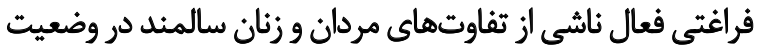
اقتصادى و اجتماعى آنهاست.

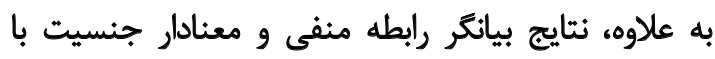

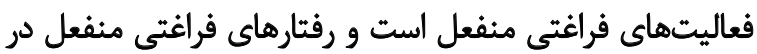
ميان زنان سالمند كمتر از مردان سالمند است.
زنان سالمند بررسى شده در مقايسه با مردان وضعيت اقتصادى

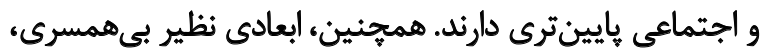

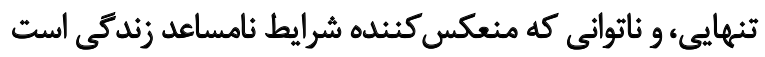

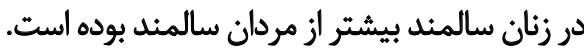

\section{تفاوتهاى جنسيتى در الكَوهاى تَّدَران وقت}

بررسى الگوهاى تذران وقت و زمان صرفشده براى هركدام

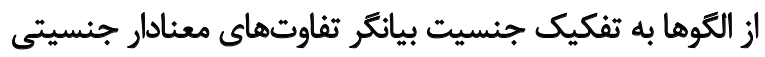

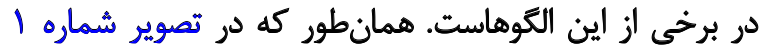

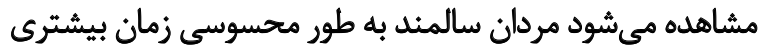

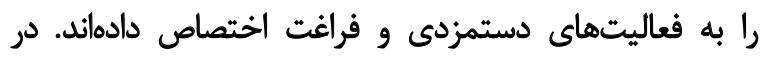

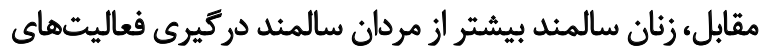
غيردستمزدى (نظير مراقب و خدمات به اعضاى خانوار مار) بودهاند. علاوه بر متوسط زمان صرفشده براى هريك از فعاليتهاي

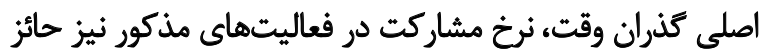

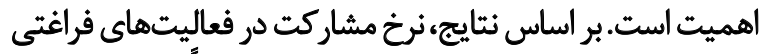

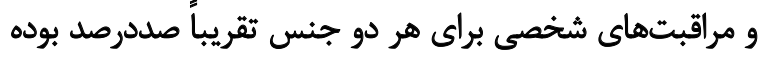

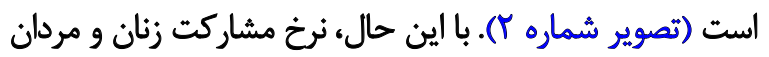

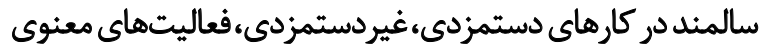

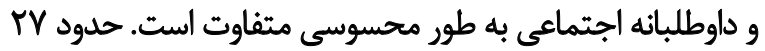

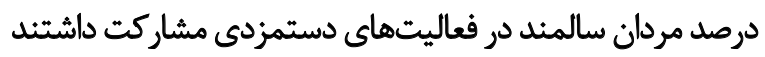

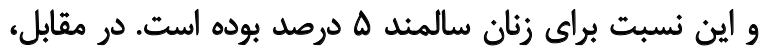

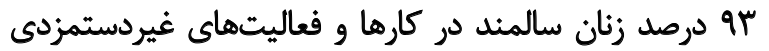

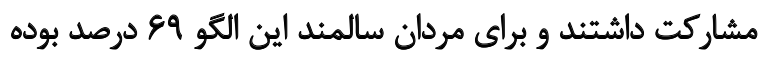

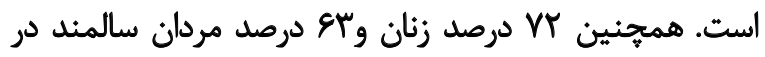

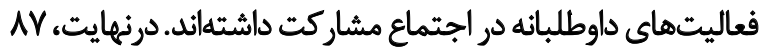
درصد زنان و Af درصد مردان سالمند در فعاليتهائ معنوى مشاركت داشتهاند.

از اين رو، نرخ مشاركت مردان سالمند در فعاليتهاى

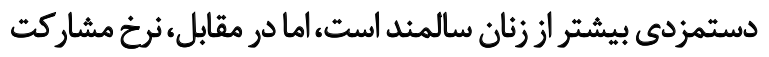

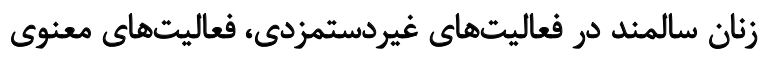

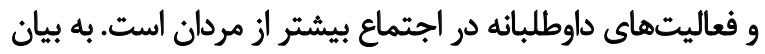

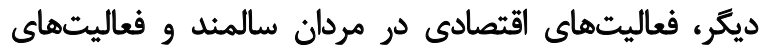

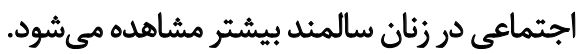

تحليل هندمتغيره

همانطور كه اشاره شد تفاوتهاى جنسيتى محسوسى در درد

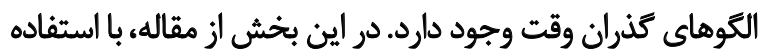

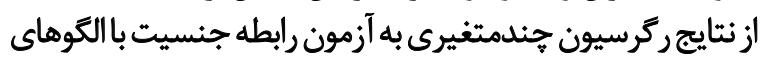

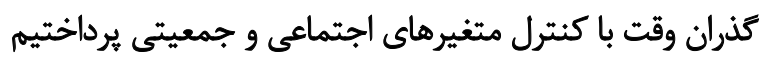

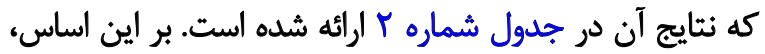

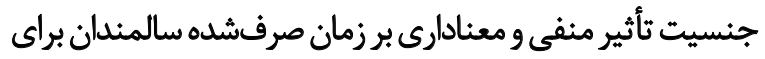

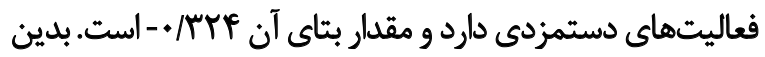


جدول r. نتايج تحليل جندمتغيره رابطه جنسيث با الكوهاى كذران وقت با كنترل متغيرهاي اجتماعى جمعيتى

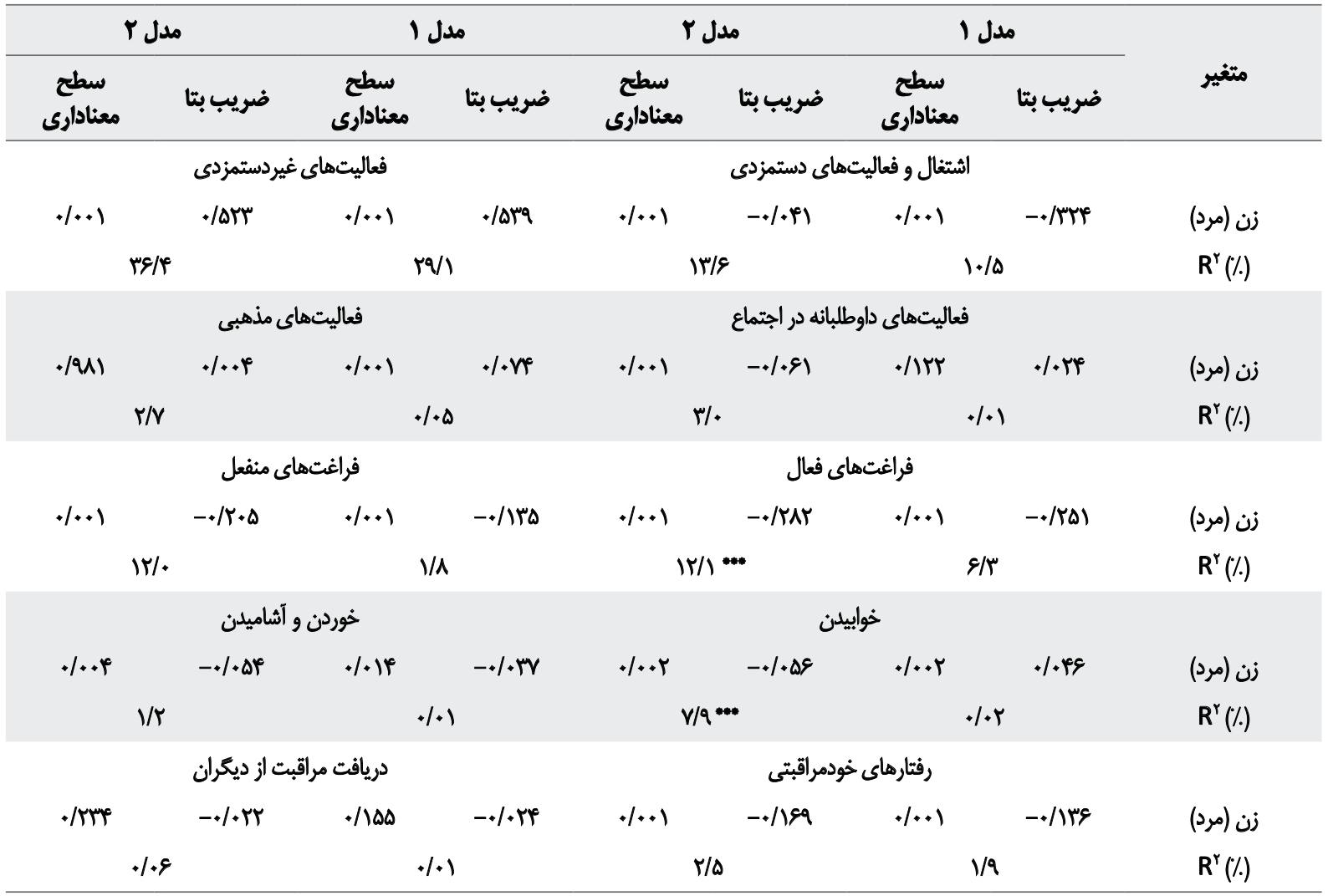

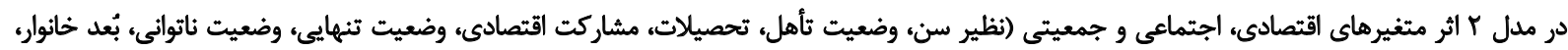

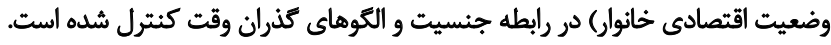

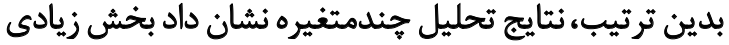

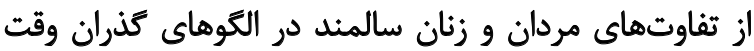

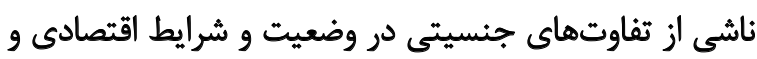
اجتماعى است.

A

جنسيت و دورانهاي مختلف زندكى الكوهاى كذران وقت و تخصيص زمان را به شيوههاي مختلف تحت تأثير قرار مي دهنئ

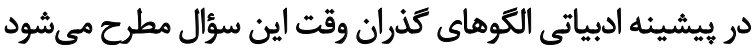

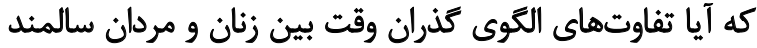

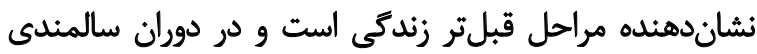

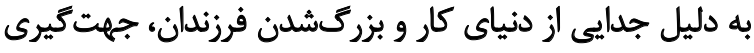

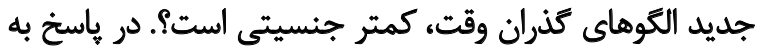
اين سؤال مطرح مى شود كه به دليل ناتوانى در دوران سالمندى

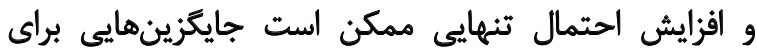

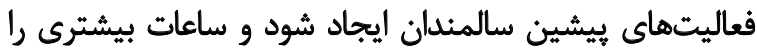

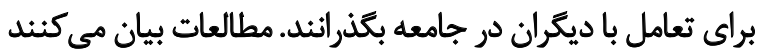

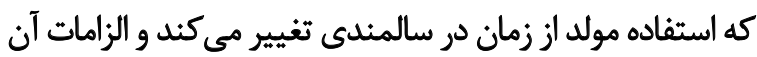

علاوه بر اينها، نتايج جدول شماره Y نُشان داد جنسيت رابطه

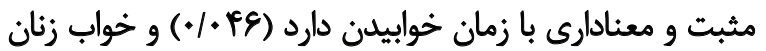

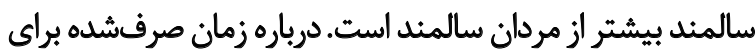

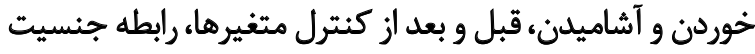

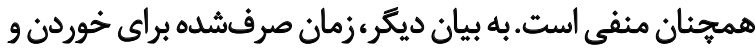
آشاميدن براى زنان سالمند كمتر از مردان سالمند است بـان درنهايت، جدول شماره † رابطه جنسيت با زمان صرفشده

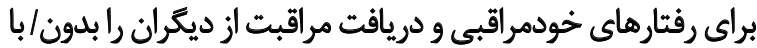

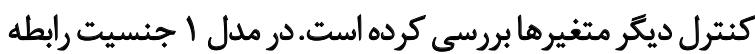

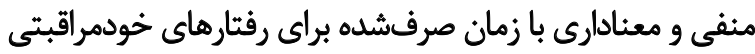

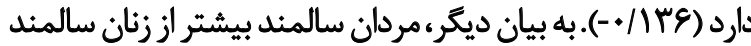

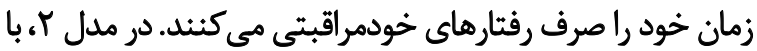
وجود كنترل ديكر متغيرهاي اقتصادى،اجتماعي و جمعيتي رابطه

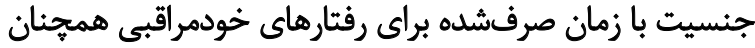

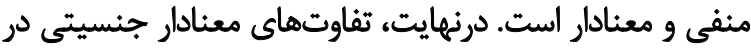

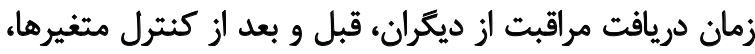

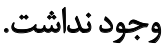


تحت تأثير تفاوتهاى جنسيتى در ابعاد اقتصادى و اجتماعى

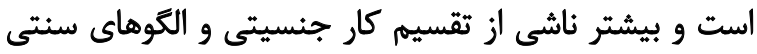

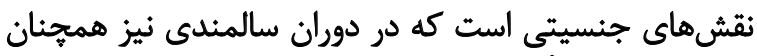

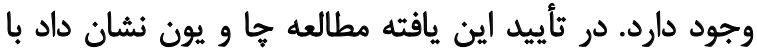

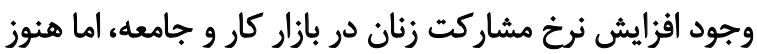

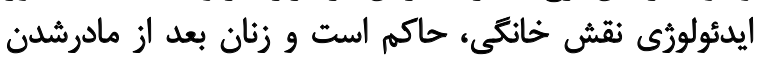

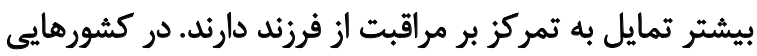

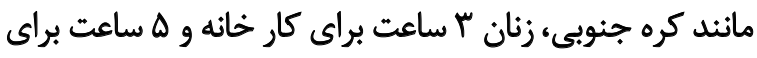

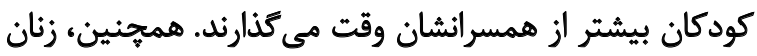

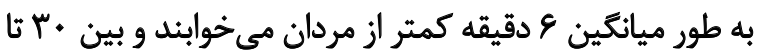

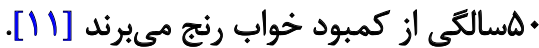

بر اساس يافتهها ميانكين زمان صرفشده زنان و مردان سالمند

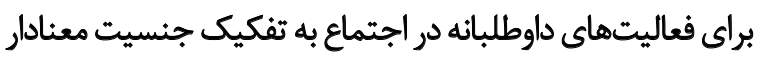

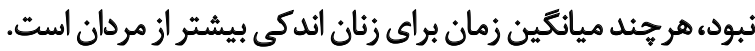

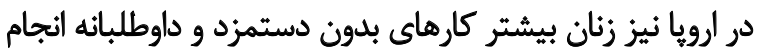

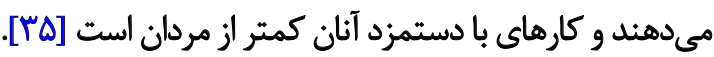
باتوجه به بافتار جامعه ايران، نرخ مشاركت زنان و مردان سالمند

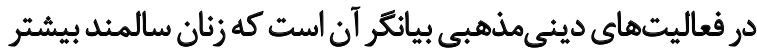

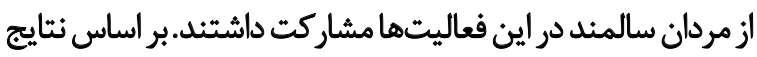

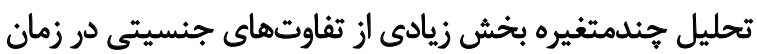

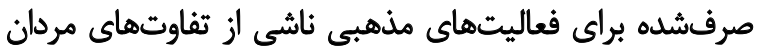

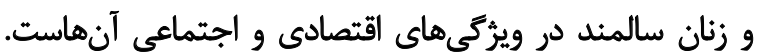

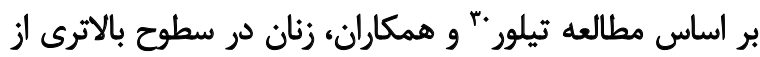

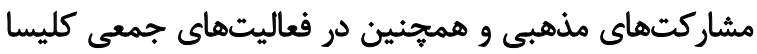

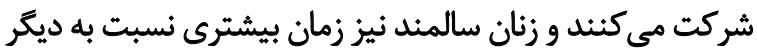

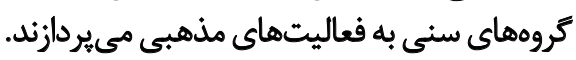

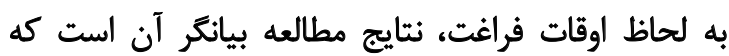

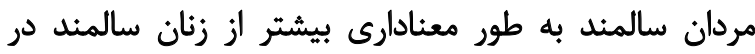

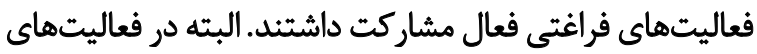

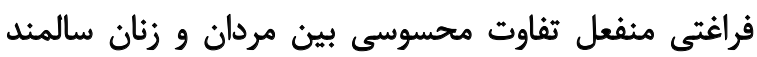

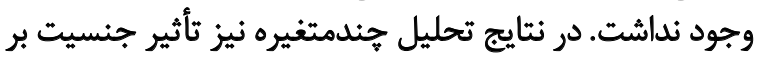

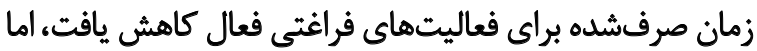

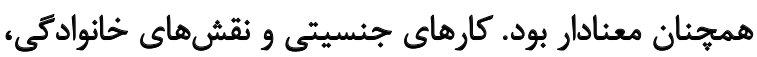

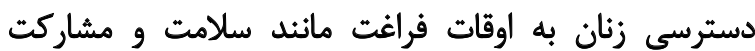

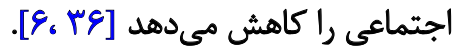

در زمينه خوابيدن، زمان خواب زنان سالمند بيشتر از مردان

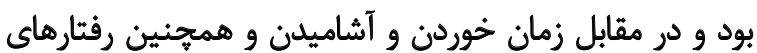

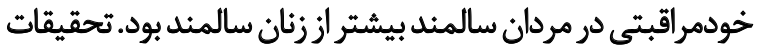

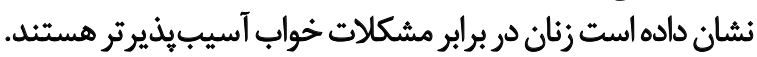

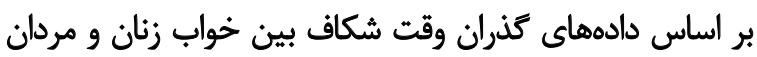

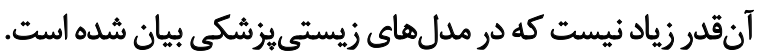

بيشتر با بذزيستن همراه مىشود. ابعاد ذهنى و عينى زمان در

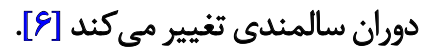

بر اساس نتايج مطالعه، سالمندان بررسى شيده بيشترين زمان

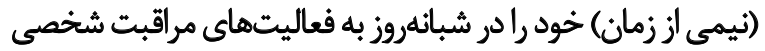

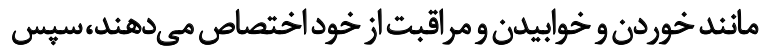

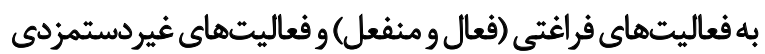

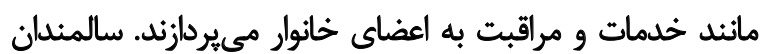

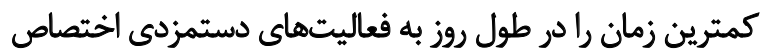

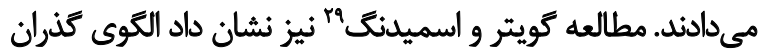

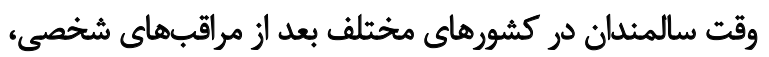
بيشتر به فعاليتهاى غيرفعالانه مانند كتاب و ورسانه اختصاص دارئ دارد.

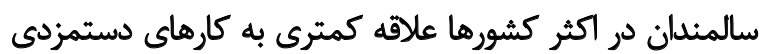

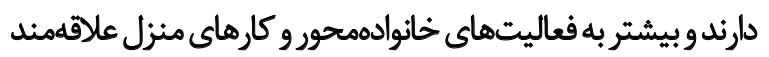

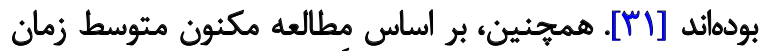

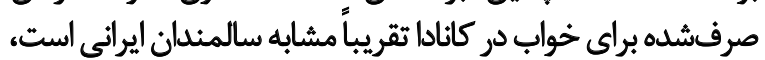

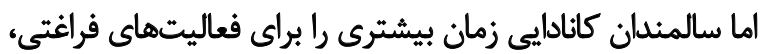
مراقبت شخصى وفعاليتهاي توليدى مي كذارنداند

نتايج درباره تفاوتهاى جنسيتى در الكوهاى تذّران وقت نشان

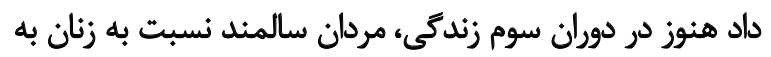

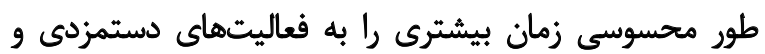

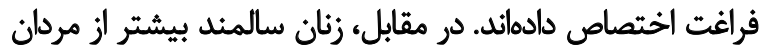

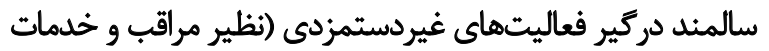

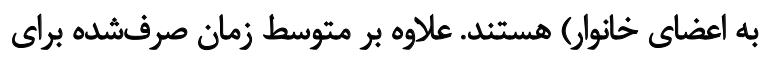

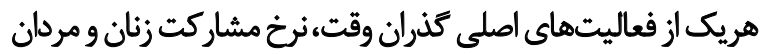

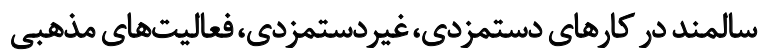

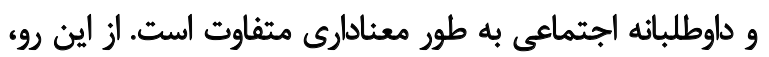

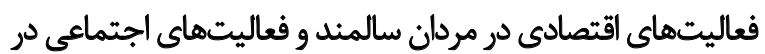

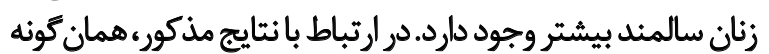

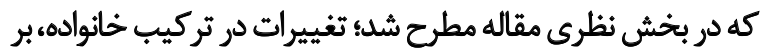

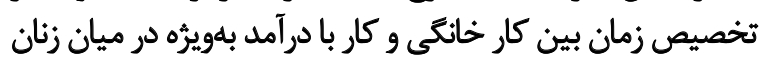

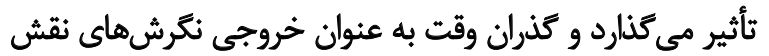

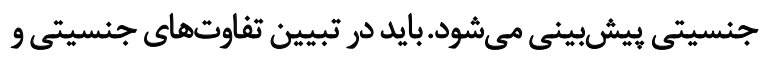

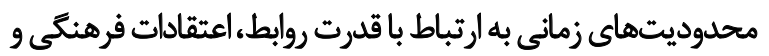

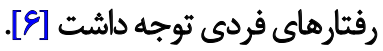

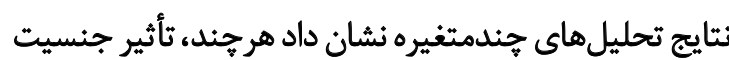

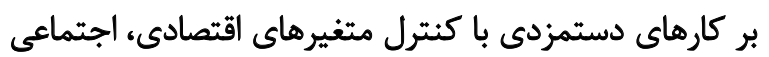

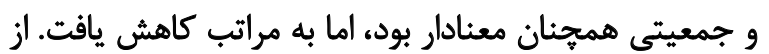

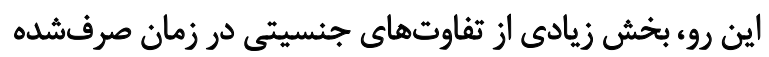

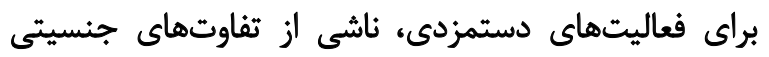

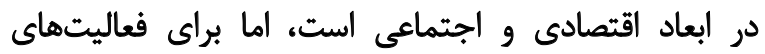
غيردستمزدى، كه در زنان سالمند بيشتر بوده است، كمثر فيترائ 
زنان)، f. موانع تحصيلى (دسترسى كمتر به آموزش و تحصيلات)

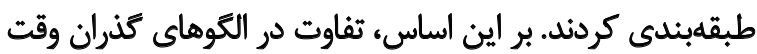

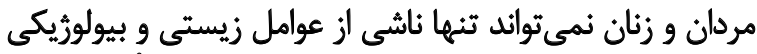

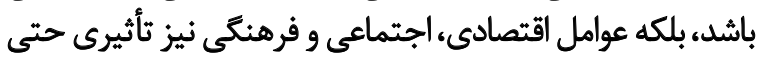

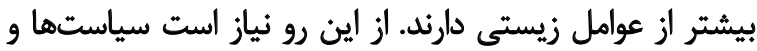

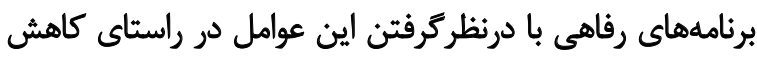

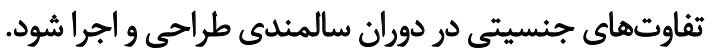
با توجه به محدوديتهاى اين مطالعه، بيشنهاد ميشود در در

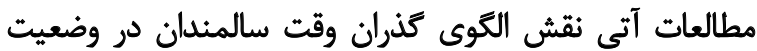

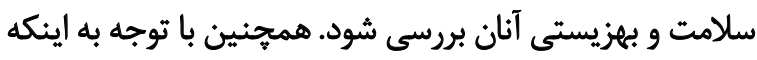

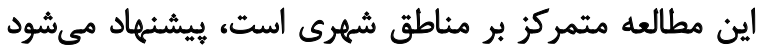

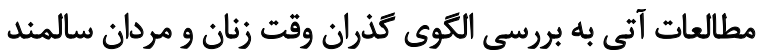
روستايى نيز بيردازند.

مالاحظات اخلاقي

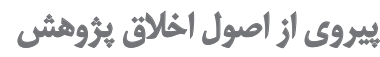

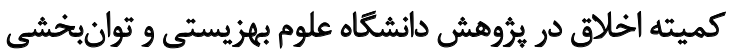

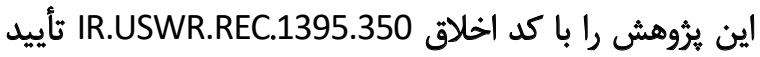

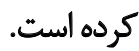

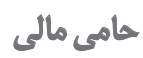

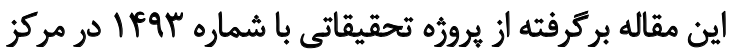

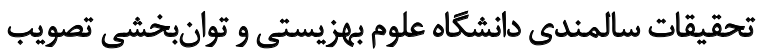
شده است.

$$
\text { مشاركت نويسندكًان }
$$

مفهومسازى و روششناسى: نسيبه زنجرى و رسول صادقي؛

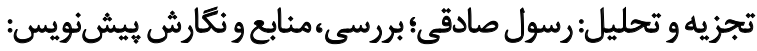

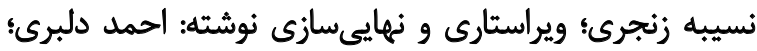

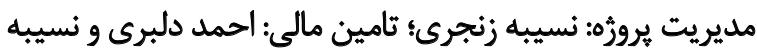

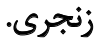

$$
\text { تمارض منافع }
$$

بنا به اظهار نويسندكان، در اين مقاله تعارض منافع وجود ندارد.

$$
\text { تشكر وقدرواني }
$$

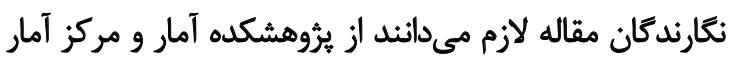
ايران براى دراختياركذاشتن دادهها تشكر و قدردانى كنئد.
كانشمندان اجتماعى بيان مى كنند كه به دليل ورود زنان به دنياى كار الكوهاى خواب آنان شبيه مردان شده است.

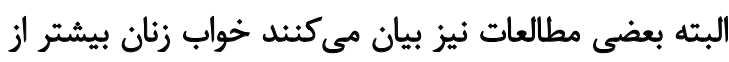

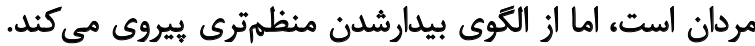

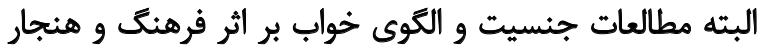

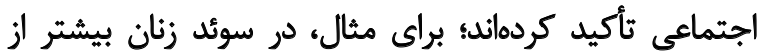

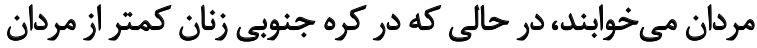

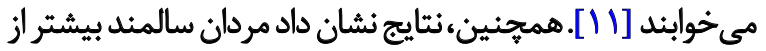

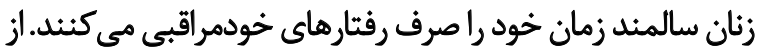

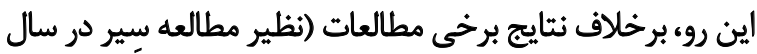

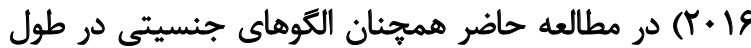

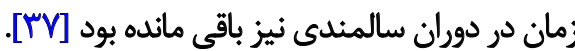

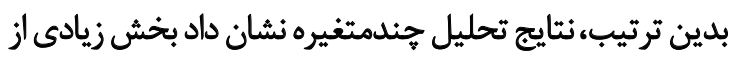

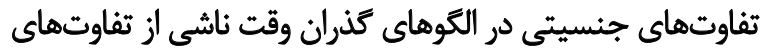

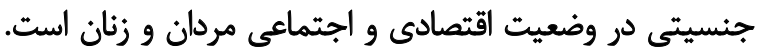

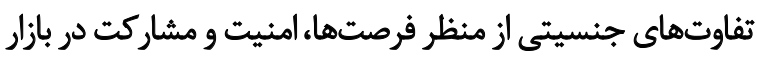

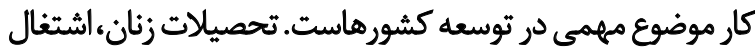

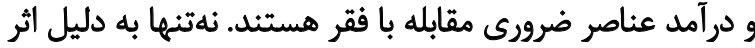

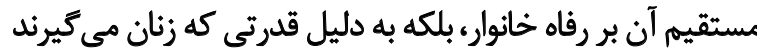

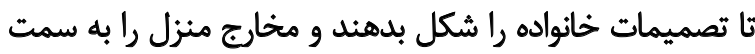

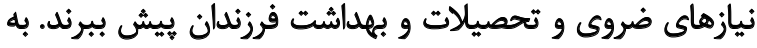

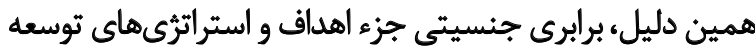

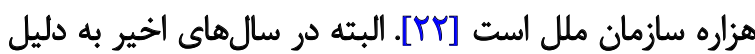

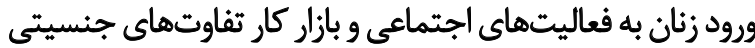

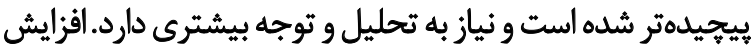

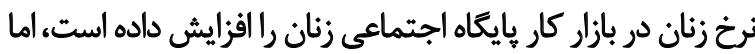

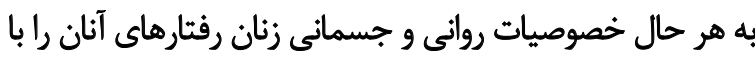

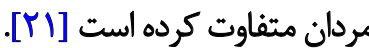

\section{نتيجلئيرى نهايى}

در تبيين تفاوتهاى جنسيتى مذكور، مطالعات علاوه بر

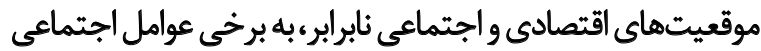

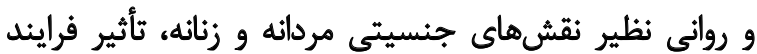

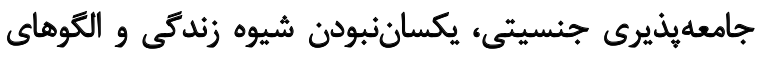

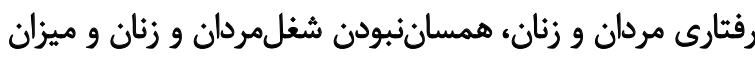

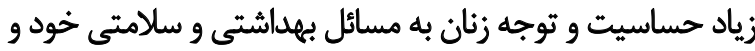
اعضاى خانوادهشان اشاره كردهاند.

در اين زمينه،اوجانغا و تيلبرت بيان مى كنند زنان در كشورهاى

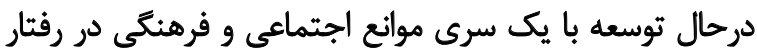

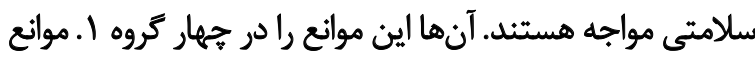

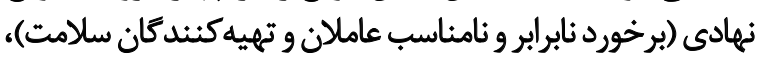

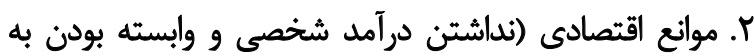

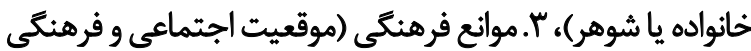




\section{References}

[1] Galay K. Patterns of Time Use and Happiness in Bhutan: Is there a relationship between the two? Tokyo: Institute of Developing Economies; 2007.

[2] Espinel PT, Chau JY, van der Ploeg HP, Merom D. Older adults' time in sedentary, light and moderate intensity activities and correlates: Application of Australian Time Use Survey. Journal of Science and Medicine in Sport. 2015; 18(2):161-6. [DOI:10.1016/j. jsams.2014.02.012] [PMID]

[3] Pruchno RA, Rose MS. Time use by frail older people in different care settings. Journal of Applied Gerontology. 2002; 21(1):523. [DOI:10.1177/0733464802021001001]

[4] Fleming R, Spellerberg A. Using Time Use Data: A history of time use surveys and uses of time use data. Wellington: Statistics New Zealand; 1999.

[5] Sadeghi R, Balali E, Mohammadpur A. [Social inequality in leisure time (Persian)]. Social Welfare. 2011; 10(39):357-89.

[6] Sayer LC, Freedman VA, Bianchi SM. Gender, time use, and aging. In: Ferraro K, George L, editors. Handbook of Aging and the Social Sciences. Amsterdam: Elsevier; 2016. [DOI:10.1016/B978-012-417235-7.00008-1]

[7] Gauthier AH, Furstenberg Jr FF. The transition to adulthood: A time use perspective. The Annals of the American Academy of Political and Social Science. 2002; 580(1):153-71. [DOI:10.1177/00 02716202580001007]

[8] Becker GS. A Theory of the Allocation of Time. The Economic Journal. 1965; 75(29):493-517. [DOI:10.2307/2228949]

[9] Flood S, Moen Ph. Time allocated to healthy behaviors among retirement-age american men and women. Minneapolis: University of Minnesota; 2011.

[10] Deem R. Women, leisure and inequality. Leisure Studies. 1982; 1(1):29-46. [DOI:10.1080/02614368200390031]

[11] Cha SE, Eun KS. Gender difference in sleep problems: Focused on time use in daily life of Korea. Social Indicators Research. 2014; 119(3):1447-65. [DOI:10.1007/s11205-013-0550-1]

[12] Muñiz C, Rodríguez P, Suárez MJ. Sports and cultural habits by gender: An application using count data models. Economic Modelling. 2014; 36:288-97. [DOI:10.1016/j.econmod.2013.09.053]

[13] Bird CE, Fremont AM. Gender, time use, and health. Journal of Health and social Behavior. 1991; 32(2):114-29. [DOI:10.2307/2137147] [PMID]

[14] McGinnity F, Russell H. Gender Inequalities in time use the distribution of caring, housework and employment among women and men in Ireland. Roscrea: Equality Authority; 2008.

[15] Piekkola H. Demographic aspects of ageing and time use in a set of European countries. Helsinki, Finland: The Research Institute of the Finnish Economy; 2004.

[16] Gray A. The changing availability of grandparents as carers and its implications for childcare policy in the UK. Journal of Social Policy. 2005; 34(4):557-77. [DOI:10.1017/S0047279405009153]

[17] Sun J. Chinese older adults taking care of grandchildren: Practices and policies for productive aging. Ageing International. 2013; 38(1):58-70. [DOI:10.1007/s12126-012-9161-4]
[18] Velarde M, Herrmann R. How retirement changes consumption and household production of food: Lessons from German time-use data. The Journal of the Economics of Ageing. 2014; 3:110. [DOI:10.1016/j.jeoa.2013.12.003]

[19] Breen R, Cooke LP. The persistence of the gendered division of domestic labour. European Sociological Review. 2005; 21(1):43-57. [DOI:10.1093/esr/jci003]

[20] Cao X, Chai Y. Gender role-based differences in time allocation: Case study of Shenzhen, China. Journal of the Transportation Research Board. 2007; 2014(1):58-66.

[21] Yang M, Li D, Wang W, Zhao J, Chen X. Modeling genderbased differences in mode choice considering time-use pattern: analysis of bicycle, public transit, and car use in suzhou, China. Advances in Mechanical Engineering. 2013; 5:706918. [DOI:10.1155/2013/706918]

[22] Habimana O, Pasqua S. Gender differences in time allocation: Evidence from Rwanda. Turin: Turin School of Development; 2017.

[23] Pedrero Nieto M. Time use and gender inequalities. Some evidence from three latin American countries. Acta Colombiana de Psicología. 2013; 16(2):55-62. [DOI:10.14718/ACP.2013.16.2.5]

[24] Maxwell NL, Wozny N. Gender gaps in time use and earnings: What's norms got to do with it? Chicago: Mathematica Policy Research; 2017.

[25] Anxo D, Mencarini L, Pailhé A, Solaz A, Tanturri ML, Flood L. Gender differences in time use over the life course in France, Italy, Sweden, and the US. Feminist Economics. 2011; 17(3):15995. [DOI:10.1080/13545701.2011.582822]

[26] Gershuny J, Sullivan O. Time use, gender, and public policy regimes. Social Politics. 2003; 10(2):205-28. [DOI:10.1093/sp/jxg012]

[27] Cutler SJ, Hendricks J. Leisure and time use across the life course. In: Binstoke RH, George LK, editors. Handbook of Aging and the Social Sciences. 1990; 3:169-85.

[28] Kellly J. Activity and ageing: challenge in retirement in work leisure and well being. Abingdon: Routledge Press; 1997.

[29] Larson R, Zuzanek J, Mannell R. Being alone versus being with people: Disengagement in the daily experience of older adults. Journal of Gerontology. 1985; 40(3):375-81. [DOI:10.1093/geronj/40.3.375] [PMID]

[30] Marcum CS. Age differences in daily social activities. Research on Aging. 2013; 35(5):612-40. [DOI:10.1177/0164027512453468] [PMID] [PMCID]

[31] Gauthier AH, Smeeding TM. Time use at older ages: Crossnational differences. Research on Aging. 2003; 25(3):247-74. [DOI:10.1177/0164027503025003003]

[32] McKinnon AL. Time use for self care, productivity, and leisure among elderly Canadians. Canadian Journal of Occupational Therapy. 1992; 59(2):102-10. [DOI:10.1177/000841749205900206]

[33] Gauthier AH, Smeeding TM. Historical trends in the patterns of time use of older adults. In: Tuljapurkar Sh, Ogawa N, Gauthier $\mathrm{AH}$, editors. Ageing in advanced industrial states. Berlin: Springer; 2010.

[34] Bediako G, Vanek J. Trial international classification of activities for time-use statistics. New York City: United Nations Statistic Division; 1998. 
[35] Bianchi S, Lesnard L, Nazio T, Raley S. Gender and time allocation of cohabiting and married women and men in France, Italy, and the United States. Demographic Research. 2014; 31:183-216. [DOI:10.4054/DemRes.2014.31.8] [PMID] [PMCID]

[36] Taylor RJ, Chatters LM, McKeever Bullard K, Wallace Jr JM, Jackson JS. Organizational religious behavior among older African Americans: Findings from the national survey of American life. Research on Aging. 2009; 31(4):440-62 [DOI:10.1177/0164027509333453] [PMID] [PMCID]

[37] Sayer LC, Freedman VA, Bianchi SM. Gender, time use, and aging. In: Binstock R, George L, Cutler S, Hendricks J, Schulz J, editors. Handbook of Aging and the Social Sciences. Cambridge: Academic Press; 2015

[38] Habimana O, Pasqua S. Gender differences in time allocation: Evidence from Rwanda. Turin: Internetional Training Center; 2017. 
This Page Intentionally Left Blank 